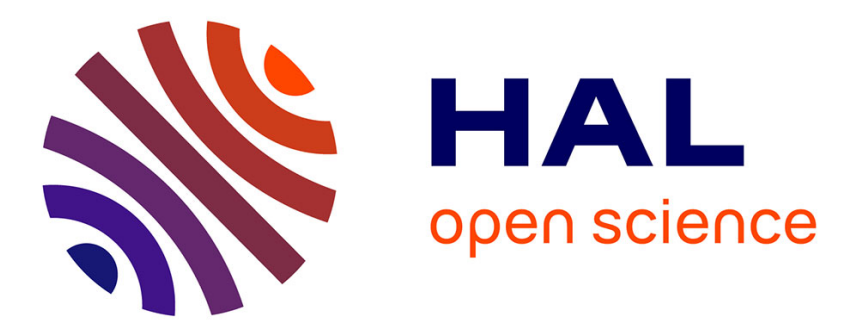

\title{
Paternal multigenerational exposure to an obesogenic diet drives epigenetic predisposition to metabolic diseases in mice
}

Georges Raad, Fabrizio Serra, Luc Martin, Marie-Alix Derieppe, Jérôme

Gilleron, Vera Costa, Didier Pisani, Ez-Zoubir Amri, Michele Trabucchi,

Valerie Grandjean

\section{To cite this version:}

Georges Raad, Fabrizio Serra, Luc Martin, Marie-Alix Derieppe, Jérôme Gilleron, et al.. Paternal multigenerational exposure to an obesogenic diet drives epigenetic predisposition to metabolic diseases in mice. eLife, 2021, 10, 10.7554/eLife.61736 . hal-03472638

\section{HAL Id: hal-03472638 \\ https://hal.science/hal-03472638}

Submitted on 9 Dec 2021

HAL is a multi-disciplinary open access archive for the deposit and dissemination of scientific research documents, whether they are published or not. The documents may come from teaching and research institutions in France or abroad, or from public or private research centers.
L'archive ouverte pluridisciplinaire HAL, est destinée au dépôt et à la diffusion de documents scientifiques de niveau recherche, publiés ou non, émanant des établissements d'enseignement et de recherche français ou étrangers, des laboratoires publics ou privés. 


\section{Paternal multigenerational exposure to an obesogenic diet drives epigenetic}

\section{2 predisposition to metabolic diseases in mice}

3 Georges Raad ${ }^{1,2,3}$, Fabrizio Serra ${ }^{1,4}$, Luc Martin $^{2}$, Marie-Alix Derieppe ${ }^{2,5}$, Jérôme Gilleron ${ }^{6}$,

4 Vera L Costa ${ }^{1}$, Didier F Pisani ${ }^{2}$, Ez-Zoubir Amri ${ }^{2}$, Michele Trabucchi ${ }^{1 \dagger}$ and Valérie

5 Grandjean $^{1 \dagger *}$

6

7

8 'Université Côte d'Azur, Inserm, C3M, Team Control of Gene Expression (10), Nice, France

$9{ }^{2}$ Université Côte d'Azur, CNRS, Inserm, iBV, Nice, France.

$10{ }^{3}$ Present address, Al-Hadi Laboratory and medical center, Beirut, Lebanon

$11{ }^{4}$ Present address, Institute for Maternal and Child Health IRCCS Burlo Garofolo, Trieste,

12 Italy

$13{ }^{5}$ Present address, Université de Bordeaux, Bât B3, Allée Geoffroy St Hilaire, CS 50023

1433615 Pessac, France

$15{ }^{6}$ Université Côte d'Azur, Inserm, C3M, Team Cellular and Molecular Pathophysiology of

16 Obesity and Diabetes (7), Nice, France

17

$18{ }^{\dagger}$ co-last author

19

$20{ }^{*}$ Correspondence to: Valérie Grandjean (Valerie.Grandjean@unice.fr). 


\section{Abstract}

23 Obesity is a growing societal scourge. Recent studies have uncovered that paternal 24 excessive weight induced by an unbalanced diet affects the metabolic health of offspring. 25 These reports mainly employed single-generation male exposure. However, the consequences of multigenerational unbalanced diet feeding on the metabolic health of progeny remain largely unknown. Here, we show that maintaining paternal western diet

28 feeding for five consecutive generations in mice induces an enhancement in fat mass and 29 related metabolic diseases over generations. Strikingly, chow-diet-fed progenies from these 30 multigenerational western-diet-fed males develop a "healthy" overweight phenotype 31 characterized by normal glucose metabolism and without fatty liver that persists for 4 32 subsequent generations. Mechanistically, sperm RNA microinjection experiments into zygotes suggest that sperm RNAs are sufficient for establishment but not for long-term

34 maintenance of epigenetic inheritance of metabolic pathologies. Progressive and permanent 35 metabolic deregulation induced by successive paternal western-diet-fed generations may 36 contribute to the worldwide epidemic of metabolic diseases.

Keywords: epigenetic, Western Diet, transgenerational inheritance, sperm, mice, RNA 
40 Nongenetic inheritance of newly acquired phenotypes is a relatively new concept in biology 41 whereby changes induced by specific environmental cues in parents (mothers and/or fathers) 42 can be transmitted to the next generations (1-3). This process is evolutionarily conserved and has been described from worms to humans (4-7). The fact that environmental cues have the potential to modify the molecular hereditary information carried by the spermatozoa demonstrates that the environmentally induced epigenetic modifications (8) are not erased through the epigenetic reprogramming process, causing them to be inherited by the next generations $(9,10)$. Although the role of epigenetic modifications such as DNA methylation $(9,11,12)$ and chromatin modifications $(13,14)$ cannot be excluded in this process, independent experimental data strongly evoke the central role of sperm RNA as a vector of paternal intergenerational epigenetic inheritance of, at least, environmentally induced metabolic pathologies $(1,3,15)$. Unlike genetic inheritance, environmentally induced epigenetic alterations are reversible, enabling the loss of previously acquired characteristics (16). Although environmental changes might persist over several generations, most reports have been based on the maintenance of paternal environmental cues for just one generation (17). This is particularly true for certain lifestyle habits, such as eating high-fat or high-sugar junk food, also called a Western diet (WD). Thus, although people around the world may face multigenerational unbalanced nutrition, there have been limited studies on its effects on the metabolic health of the progeny.

Herein, we studied the impact of the paternal maintenance of an unhealthy WD for multiple generations on the metabolic phenotype of both the progenitors and their respective chow61 diet-fed (CD-fed) offspring. 


\section{Results}

\section{Feeding successive paternal generations with a western diet exacerbates the} overweight phenotype and accelerates the development of obesityassociated pathologies

To test experimentally whether the maintenance of an unhealthy diet through the paternal germline influences the metabolic phenotype of the resulting individuals, C57BL6/J male mice were fed a WD for five consecutive generations (from WD1 to WD5) (Figure 1A and Figure 1-figure supplement $1 \mathrm{~A})$. According to a previous study (18), multigenerational WD feeding exacerbates the increased body weight mass induced by this diet. Despite marked heterogeneity in the WD4 and WD5 populations, we found that the WD4 and WD5 males weighted significantly more than the WD1 and WD3 ones ( $p<0.05$ and $p<0.01$, respectively) (Figure 1B and Figure 1-figure supplement 2A, B). Interestingly, growing heterogeneity of the body weight mass between the males of the first and the latter generations (Figure 1-figure supplement 1C) was observed in the 4 independent families, indicating that the phenotypic heterogeneity previously observed in diet-induced obesity mouse models (19) increases progressively over the generations. This increase in total body weight with paternal multigenerational WD feeding was associated with an increase in perigonadal white adipose tissue (gWAT) mass (Figure 1C and Figure 1-source data 1). Indeed, WD5 gWAT weighted significantly more than the WD1 gWAT $(p<0.05)$ and the gWAT volume measured by computed tomography increased 2.3-fold and 3.4-fold in WD1 and WD5 mice, respectively, compared to that of control mice (CD-fed mice) (Figure 1-source data 1). The increase in gWAT mass was positively correlated with total body weight (perigonadal fat mass versus total body weight; Spearman's $r=0.78, p<0.0001$ ) (Figure 1-figure supplement 2E). It was also associated with the hypertrophy of white adipocytes, with a median surface cell area of white adipocytes increasing from 1500 to $4000 \mu \mathrm{m}^{2}$ from the first (WD1) to the fifth generation (WD5) and with a decreased calculated number of adipocytes in WD5 compared 
to the controls (Figure 1D-F). Furthermore, our RNA-seq comparison between the gWAT of WD1 and WD5 males revealed that multigenerational WD feeding has a strong impact on the gWAT gene expression profile. In fact, we observed an increase in differentially expressed genes (DEGs), from 325 in WD1 (with 93 upregulated and 232 downregulated genes) to 1199 (757 upregulated and 442 downregulated) in WD5, compared to the respective CD-fed mice. Interestingly, while the majority of DEGs in WD1 (66\%) were also deregulated in WD5, a minority of DEGs in WD5 (only $8 \%$ for the upregulated genes and $35 \%$ for the downregulated genes) were deregulated in WD1 ( $p$ value<0.01). Importantly, all common genes were deregulated in the same direction (Figure 1G). Interestingly, querying the WD1 and WD5 DEGs against the molecular signature database (MSigDB) collection of curated gene pathway annotations revealed a specific WD5 enrichment in gene sets associated with

100 CHEN_METABOLIC_SYNDROM_NETWORK (genes forming the macrophage-enriched 101 metabolic network (MEMN) claimed to have a causal relationship with metabolic syndrome 102 traits) and with genes potentially regulated by the methylation of lysine 4 (H3K4) and lysine 10327 (H3K27) of histone H3 and by polycomb repressive complex 2 (PRC2) (Figure 1-source 104 data 2) (20).

105 The aforementioned modulations of white adipose tissue in WD generations shed light on the possible exacerbation of obesity-associated pathologies (such as insulin resistance (and

107 subsequently type-II diabetes) and nonalcoholic fatty liver disease) (21). To check this 108 hypothesis, several metabolic risk parameters related to these pathologies were analyzed in 109 WD-fed mice (Table 1). In comparison with CD-fed mice, circulating plasma levels of leptin, 110 C-reactive protein (CRP), one marker of inflammation, and total cholesterol were significantly 111 higher in the WD3 $(p<0.01)$, WD4 $(p<0.05)$ and WD5 $(p<0.01)$ groups but not in the WD1 $112(p=0.07)$ or WD2 $(p=0.4)$ groups (Table 1$)$. The alterations in these metabolic parameters in

113 WD-fed males were found to be positively correlated with the increase in gWAT mass (Figure

114 1-figure supplement 2F-H). At the molecular level, the increase in serum leptin in WD-fed 115 males was positively correlated with an increase in leptin mRNA levels in the gWAT of the 116 respective male mice (total serum leptin and leptin mRNA, Spearman's $r=0.89, p<0.0001$, 
117 Figure 1-figure supplement 2I), suggesting an accumulation of epigenetic modifications at the

118 leptin promoter. These results are in line with recent studies showing that leptin upregulation 119 occurs via epigenetic malprogramming in white adipose tissue $(22,23)$. Furthermore, we 120 found a significantly impaired response in the intraperitoneal glucose tolerance test (GTT) in 121 all WD-fed mouse groups (Figure 2A), which was not associated, except for in WD2-fed 122 males, with an impaired insulin response, as shown by the intraperitoneal insulin tolerance 123 test (ITT) (Figure 2B). Therefore, unlike the other metabolic parameters, we did not notice 124 any significant exacerbation of insulin sensitivity in successive generations. Moreover, the 125 response to an intraperitoneal glucose tolerance test (measured through the AUC-GTT 126 calculation) was not correlated with the gWAT mass (Figure 1-figure supplement 2J). 127 Together, these data might reflect the multifactorial and complex nature of the pathogenesis 128 of obesity-induced diabetes.

129 Strikingly, although the C57BL6/J-strain male mice fed a WD diet for one generation failed to

130 develop strong alterations in liver phenotype $(24,25)$, major abnormalities were observed in 131 WD5 liver, i.e., organ weight, histological and biochemical parameters. Indeed, the mass of 132 the WD5 liver (not that of the WD1 liver) was significantly higher than that of the CD 133 specimens (Figure 2C). Furthermore, unlike WD1 liver, histological and biochemical 134 examinations revealed the presence of macrovesicular steatosis with significantly increased 135 triglyceride (TG) levels in WD5 liver compared with CD liver ( $p<0.01$, respectively) (Figure 136 2D-F). Therefore, the phenotype of WD5 livers exhibits typical features of fatty liver.

137 Together, both morphological and molecular features demonstrate that multigenerational WD 138 feeding induced a progressive dysregulation of the male metabolic phenotypes (Figure 1139 figure supplement $2 \mathrm{~K}$ ), with an exacerbation of the gWAT size and gWAT transcriptional 140 alteration as well as of obesity-associated pathologies such as fatty liver. Therefore, a 141 worsening of the underlying medical conditions can be potentially transmitted to next 142 generations. 

phenotype

145 Previous reports showed that WD-induced metabolic dysregulations during one-generation exposure could be transmitted across 1 (F1) or 2 generations (F2) fed a CD $(2,11)$. To investigate the impact of feeding a WD through several generations on the inheritance of

148 diet-induced metabolic pathologies, we compared the metabolic status of F1, F2 and F3

149 cohorts fed a CD generated from either CD, WD1 or WD5 males (Figure 3A). As expected

150 from previous studies (2-3), male and female F1 progenies derived from WD1 males (F1-

151 WD1) were statistically heavier than the control animals with CD-fed ancestors (Figure 3B, F

152 and Figure 3-figure supplement $1 \mathrm{~A}-\mathrm{C})$. Although the difference did not reach significance at

153 the age of 16 weeks, the same trend was also observed for the $\mathrm{F} 1$ progenies derived from 154 WD5 (F1-WD5) male progenies (Figure 3D,H and Figure 3-figure supplement 1A-C). This 155 overweight phenotype was associated with impaired glucose tolerance as measured by the

156 GTT for both the male F1-WD1 and F1-WD5 progenies and the female F1-WD5 mice (Figure

157 3-figure-supplement 1E,G and Figure 3-source data 1,2). We noticed, however, the absence 158 of intergenerational inheritance of the fatty liver phenotype observed in the WD1 and WD5 159 progenitors (Figure 3-figure supplement 2).

160 Both male F2-WD1 and F2-WD5 CD-fed progenies were also overweight $(p<0.01)$. This 161 phenotype was associated with an excessive accruement of gWAT mass of at least $90 \%$ 162 over the control (Figure 3 C,E,G,I). Importantly, although the female and male F2-WD5 163 progenies were found to be significantly fatter and heavier than the F2-WD1 cohorts, these 164 mice did not exhibit impaired glucose tolerance (as measured by the GTT) (Figure 3-figure 165 supplement 1E,G) or signs of fatty liver lesions (Figure 3-figure supplement 2).

166 The metabolic differences were even more striking in both F3 and F4 progenies (Figure 3 167 and Figure 3-figure supplement 1). Thus, as illustrated in Figure 3B-I, the populations of males and females of the F3-WD1 progenies were very homogeneous, exhibiting metabolic 
169 characteristics very similar to control mice. By contrast, both populations of males and

170 females of the F3-WD5 progenies were heterogeneous in terms of body and gWAT weights, 171 some of them showing weights closed to CD mice and others being clearly overweight and 172 fat. However, both F3-WD5 populations were significantly heavier and fatter $(\mathrm{p}<0.001$ and $173 \mathrm{p}<0.01$, respectively) than control and F3-WD1 populations (Figure 3B-I and Figure 3-source

174 data 1,2). Strikingly, despite being overweight, the progenies derived from WD5-fed animals 175 did not display any alterations in terms of glucose metabolism (Figure 3-figure supplement $1761 \mathrm{E}, \mathrm{F}, \mathrm{G}, \mathrm{H}$ ) and fatty liver pathologies at 4 months of age (Figure 3-figure supplement 2 and

177 Figure 3-source data 1,2).

178 Collectively, these data suggest that WD feeding for multiple generations induces stable 179 germline epigenetic modifications that were not erased after removing the stressor(s) for at 180 least 4 generations of CD-fed progeny.

181 Sperm RNAs transmit only transient epigenetic inheritance of WD-induced pathologies

183 Specific signatures of sperm small non-coding RNAs (sncRNAs) from WD-fed mice have

184 been previously shown to act as a vector of intergenerational epigenetic inheritance of newly 185 acquired pathologies $(1,3,15,26)$. To determine whether sperm small RNAs are also 186 involved in the long-term maintenance of epigenetic inheritance (transgenerational epigenetic 187 inheritance), we systematically profiled the expression of small non-coding RNAs of 3 188 independent CD, WD1 and WD5 sperm samples by deep sequencing using a recently 189 developed sncRNA annotation pipeline optimized for rRNA- and tRNA-derived sRNAs, 190 rsRNA and tsRNA, respectively (SPORTS1.0) (27). We found a sperm RNA signature that is

191 specifically induced in the first generation of WD-diet males characterized by an increased in 192 the proportion of rsRNA. This is consistent with previous reports showing the sensitivity of 193 rsRNA to dietary exposure such as an High Fat Diet (HFD) $(28,29)$ (Figure 4B). Strikingly, 194 this sncRNA signature observed in the first generation of WD-fed male fed a WD tends to 
disappear in the fifth generation of WD-fed male which exhibit a sncRNAs signature very similar to that of CD sncRNAs. On the one hand, while $\sim 64 \%$ of the sncRNAs were annotated to rsRNAs in WD1, only $\sim 40 \%$ of them were annotated to rsRNAs in CD and WD5 sperm (Figure 4D), suggesting a global modification of the sncRNA population upon WD exposure. On the other hand, a closer look at the sncRNAs differentially expressed in WDs (WD1 and WD5) and CD showed that the rsRNAs are not the only population to be sensitive to dietary exposure (Figure 4), which is consistent with previous reports in mice. Indeed, searching for small RNA DEGs (adjusted $p$ value $<0.05$ ) between WD (WD1 or WD5) and CD sperm, we identified 479 and 66 DEGs in WD1 and WD5, respectively, compared to control mice (Figure 4E and Figure 4-source data 1, 2). Interestingly, the majority of WD5 DEGs (47 out of 66) were already deregulated in WD1 (Figure 4E). Among these common sncRNAs, we identified tsRNA and microRNAs known to be involved in short-term epigenetic inheritance of metabolic dysfunction (intergenerational inheritance) $(1,3)$ (Figure 4F and Figure 4-source data 1, 2). Interestingly, no sncRNA was found deregulated (adjusted $\mathrm{p}<0.05)$ between WD1 and WD5. These data indicate that the sensitivity of sperm sncRNA signature to diet, observed independently by several group $(28,29)$, is also modulated by the diet of ancestors.

212 To further investigate the role of sperm RNAs in the long-term transgenerational epigenetic 213 inheritance of metabolic alterations, microinjection experiments into naïve zygotes were 214 performed with total sperm RNA from either WD1 or WD5 males (RNA-WD1 progenies and 215 RNA-WD5 progenies, respectively) (Figure 5A). As previously reported, this experiment 216 faithfully reproduces the pattern of short-term paternal transmission of environmentally 217 induced phenotypes in crosses $(1,3,4,15,26)$. In agreement with previous studies, male 218 12-week F1-RNA-WD1 and F1-RNA-WD5 progenies were heavier than F1-RNA-CD 219 progenies (31 g vs $30 \mathrm{~g}, \mathrm{p}<0.05$ ) (Figure 5B). In addition, they displayed glucose and insulin 220 response alterations, as shown by GTT and ITT analyses, with significantly higher values of 221 the area under the curve (AUC) to controls (Figure 5D,E and Figure 5-source data 1). 
222 Regarding the fatty liver phenotype, neither abnormal TG levels nor histological 223 abnormalities were observed in liver from F1-RNA-CD and F1-RNA-WD progenies. Thus, the 224 metabolic alterations observed in F1-RNA progenies are partially reminiscent of the WD1 225 and WD5 male phenotype.

226 Overweight phenotypes and glucose response alterations were partially transmitted to the F2 227 and F3 generations (Figure 5-source data 2, 3). Indeed, total body weight mass of the F2228 RNA-WD males was significantly heavier than that of the F2-RNA-CD male $(p<0.05)$. This 229 metabolic abnormality did not persist in the F3 and F4 progenies. Strikingly, although the 230 glucose and insulin response alterations were not observed in the F2 generations of RNA231 WD males, we noticed an alteration in those responses in the F3 generations of the RNA232 WD5 males but not of the RNA-WD1 males. Intriguingly, while we did not observe any liver 233 abnormalities in F1-RNA progenies, liver histological examinations revealed macro- and 234 microvesicular steatosis in hepatocytes of two F2-WD overweight males (2 out of 10) (Figure 235 5-figure supplement 1). It should be noted that these abnormal hepatocytes were never 236 observed in RNA-CD progenies. Nevertheless, all the metabolic alterations were completely 237 absent in the F4 generations (Figure 5-source data 4).

238 Thus, the metabolic observed phenotype of WD1 and WD5 progenies obtained by either 239 RNA microinjection or natural mating exhibited some discrepancies. First, the overweight 240 phenotype induced by sperm RNA from WD5 males was not exacerbated compared to that 241 induced by sperm RNA from WD1 males. In fact, no statistically significant difference was 242 observed among the body weight of the F1, F2 and F3 progenies derived from sperm RNA of 243 WD1- and WD5 animals. Second, the sperm-RNA-induced overweight phenotype was 244 associated with glucose metabolic alterations (total body weight and AUC-GTT, Spearman's $245 r=0.4, p<0.01$, Figure $5 \mathrm{~F}$ ) and was sporadically associated with fatty liver abnormalities, in 246 both WD1 and WD5 (Figure 5-figure supplement 1). Taken together, these data strongly 247 suggest that sperm RNAs can unequivocally induce intergenerational phenotype but may 248 induce some transgenerational features - although the effect is weaker than the effect 249 induced by whole sperm. This is in line with previous study using a psychological-stress 
induced model showing that sperm RNA injection can also induce phenotype in both F1 and

251 F2 generations (4). Last, in contrast to natural mating of WD5, the sperm-RNA-induced 252 overweight phenotype was not inherited for more than 2 generations. Together these results 253 indicate that sperm RNAs are not sufficient for the long-term epigenetic inheritance of 254 metabolic dysfunctions.

\section{Discussion}

256 Growing evidence suggests that an unbalanced diet of the father negatively affects its 257 metabolic health and that of its progenies. Of particular interest, little attention has been 258 focused on the effect of paternal successive generations of unbalanced diet exposure on 259 metabolic health, which may have public health and economic impacts. To this end, we fed 260 male mice for 5 successive generations on a high-fat, high-sugar diet (western diet, WD) to 261 compare the metabolic parameters across multiple generations of WD males and to assess 262 the persistence of the WD-induced metabolic alterations in their subsequent balanced CD263 fed progenies.

264 In summary, our findings reveal that maintaining a WD for several generations promotes a 265 progressive accumulation of epigenetic alterations in somatic and germ cells throughout 266 generations. Two lines of evidence support this conclusion. First, ancestral exposure 267 influences the magnitude of the overweight phenotype. Indeed, a male whose father, 268 grandfather, great grandfather, great-great-grandfather and great-great-great grandfather, up 269 to 5 generations of exposure, have been fed a WD exhibits the most severe overweight 270 phenotype associated with serious metabolic alterations. Second, the father's ancestral 271 history (whether his ancestors were fed an unbalanced diet) affected the pattern of 272 inheritance of this metabolic pathology. Second, the father's ancestral history (whether or not 273 the ancestors were fed an unbalanced diet) affected the pattern of inheritance of metabolic 274 pathologies.

275 The main limitation of our study is the phenotypic heterogeneity observed in the males of the 276 WD4 and WD5 generations (Figure 1-figure supplement 1C) and in the CD-fed WD5 277 progenies (Figure 3) which might lead to biased conclusions. Indeed, although the statistical 
278 tests we used here should rule out this weakness, we cannot rule out the possibility of the

279 presence of sub-populations. Metabolic heterogeneity induced by an unbalanced diet has

280 already been reported in mice $(19,30)$ and the strong heterogeneity observed in our model 281 may indicate an adaptative process whereby different subpopulations could emerge in 282 response to the maintenance of an unbalanced diet.

283 Although it is well described that the development of type 2 diabetes is positively associated 284 with body weight (31), we did not observe a strong correlation between fat mass and glucose 285 and insulin sensitivities in males obtained after multigenerational WD feeding. However, we 286 identified one obese-associated pathology that increased in severity with successive 287 generations of a WD, namely, hepatic steatosis, indicating that exposure sensitivity is 288 heightened by multiple generations of exposure, at least for this diet-associated pathology. 289 Thus, the family food environment, parental dietary behaviors and family obesity might be an 290 additional clue to explain the increasing incidence of nonalcoholic fatty liver disease in 291 humans (32).

292 Importantly, multiple generations of WD exposure impact not only the sensitivity to a WD but 293 also the hereditary makeup, also called background. Indeed, when the father has no WD-fed 294 ancestor, the fatness of its progenies tends to disappear after WD removal. However, in the case of fathers with several WD-fed ancestors, the progenies will remain stably overweight 296 for more than 4 generations. Intriguingly, although the male progenies of the third and fourth 297 generations of WD5 males were overweight, they did not develop metabolic alterations, such 298 as glucose/insulin sensitivity alterations and fatty liver diseases. Together, these data 299 strongly suggest that the combination of ancestral and individual diet exposure was both 300 necessary and sufficient to elicit the most severe metabolic effects in mice. Thus, it looks like 301 the five-generational western-diet-fed males have evolved to develop a protective 302 mechanism in glucose and liver fat metabolism that can be inherited by the offspring.

303 Overall, our findings are in agreement with those of recent studies of multigenerational 304 exposure performed in several animal models. For instance, in guppies, a wide range of 305 plastic responses under different light conditions were observed, which were dependent on 
306 multigenerational exposure to different light environments (33). In mites, zinc element 307 sensitivity increased by continuous multigenerational exposure (34). In mice, male sensitivity 308 to environmental estrogens was enhanced by successive generations of exposure (35). 309 Finally, rats undernourished for 50 generations showed multiple metabolic alterations that 310 were not reversed in their respective F1 and F2 CD-fed progenies (36). Together, the 311 present and previously published studies indicate that the exacerbation of stress-induced 312 phenotypes upon multigeneration exposure as well as the stabilization of newly induced 313 phenotypes is an evolutionarily conserved process. The underlying mechanism of this 314 process is intriguing and worth to be explored in the future using this model since it might 315 have not only social-medical implications, but also evolutionary perspectives.

316 Single-generation exposure to unhealthy diet strongly indicates that sperm RNAs are a 317 possible epigenetic vector of intergenerational and transgenerational epigenetic inheritance 318 of metabolic diseases $(1,3,15)$. However, these data do not exclude the possible 319 involvement of epigenetic modifications, namely, DNA methylation, histone modifications and 320 chromatin structure alterations. This study takes a step further in this direction. Indeed, on 321 the one hand, we showed that small non-coding RNAs signature is not only the reflect of the 322 diet of the father, as already demonstrated $(28,29)$ but also that of the diet of the ancestors 323 (Figure 4). Whether this is associated to spermatic epigenetic changes such as DNA 324 methylation and chromatin structure alterations is an opened question. On the other hand, 325 our microinjection experiments showed that small RNAs are vectors of intergenerational 326 inheritance but are not sufficient for the long-term inheritance of diet-induced metabolic 327 alterations. In this context, our transcriptome profile of gWAT may provide important avenues 328 to dissect the potential molecular mechanism(s) involved in this process, revealing an 329 enrichment in genes potentially regulated by H3K4/K27 methylation and the PRC2 complex 330 (Figure 1-source data 2).

331 Finally, in the present study, we focused our analyses on perigonadal adipose tissue, 332 glucose/insulin sensitivity and liver alterations. Considering the healthy and economic 333 consequences of obesity and its comorbidities, such as cardiovascular diseases and fertility 
334 abnormalities, future studies will be important to determine the impact of multigenerational

335 ancestor exposure on the development of obesity-associated comorbidities.

336 In conclusion, environmentally induced epigenetic modifications in germlines would 337 contribute to the environmental adaptation and evolution of animal species. In the future, it 338 will be important to assess how each epigenetic vector for inheritance interacts together to 339 modulate the embryonic epigenome.

\section{$340 \quad$ Materials and Methods}

$341 \quad$ Mice

342 All mouse experiments were performed with C57BL/6J mice obtained from Charles River 343 (Charles River Laboratories, France). All mice were housed in a temperature-controlled 344 system and maintained on a 12-h light/dark cycle (lights on at 7 a.m.). Experimental mice were given ad libitum access to either a high-fat high-sugar diet (WD) (U8954 version205 HF $45 \%$ of energy from fat, SAFE, France) or a control diet (CD) (SAFE A04, $5 \%$ of energy from fat, SAFE, France) and sterile water. To evaluate the impact of the diet of paternal ancestors on metabolic health, we developed two experimental models. On the one hand, WD feeding 349 was maintained for 5 successive generations through the paternal line. Briefly, 3-week-old male mice were divided into 2 groups. Males from the first group were kept on CD, and the males of the other group were fed a WD for 3 months. This first generation of CD-fed and WD-fed males was named CD1 and WD1, respectively. At four months old, 2 independent

353 males of WD1 group were then crossed with 7-week-old C57BL/6J female mice (CD-fed)

354 obtained from Charles River (Charles River Laboratories, France) to generate WD2 offspring.

355 One or two litters were obtained per male. The male progenies were kept and subjected to 356 the same experimental procedure. Then, at least one male, selected randomly, fed a WD for 3573 months from each family was mated with CD-fed females to generate WD3 offspring. The same procedure was repeated twice to generate the WD4 and WD5 offspring (Figure 1A-

359 figure supplement 1A). However, from the WD2 generation, a considerable heterogeneity 360 with respect to total body weight mass was observed within the same litter (Figure 1, figure 
supplement $1 \mathrm{C})$. For this reason and as illustrate in Figure 1-figure supplement $1 \mathrm{~A}$ starting

362 from this generation, more than one male per litter was chosen to mate. The complete experimental design was performed twice at approximately 6 months' interval giving rise to 4 independent families. To demonstrate that we didn't create significance with increasing the correlated sample, we selected a subset of 18 mice in WD4 and WD5 making sure that the 4 WD1 ancestors were equitably represented across the groups. Three combinations of 18 mice were selected and gave the same significant level with a $p$ value $<0.001$ when total body weight mass or gWAT weight relative to total body weight of WD4 and WD5 groups was compared to the corresponding weight of CD group. One of these combinations is showed in Figure 1-figure supplement 2B. The same procedure was performed for the CDgroup (Figure 1-figure supplement 1B). However, in accordance with previous studies (16, $37218,37,38$ ), body mass and gWAT weights of the CD-fed male progenies were very 373 homogeneous (Figure 1-figure supplement 2D) and were combined in the same group.

374 Then, to determine whether the WD1 and WD5 phenotypes were paternally inter- and transgenerationally inherited in absence of WD, 4 WD1 and 9 WD5 males were crossed with a CD-fed female and their respective male and female progenies were fed a CD. The first generation was called F1-WD1 and F1-WD5, respectively. The F1 4-month-old male progenies were crossed with 7-week-old C57BL/6J female mice (CD-fed) to obtain the F2WD1 and F2-WD5 progenies (Figure 3A). This experimental design was repeated once to 380 obtain the F3-WD1 and F3-WD5 progenies. The control group of this experiment was obtained by crossing CD-fed males with 7-week-old C57BL/6J female mice (CD-fed) and 382 maintained for 4 generations on CD.

383 To evaluate the role of sperm RNAs in transgenerational epigenetic inheritance of metabolic 384 alterations, sperm RNAs extracted from 2 different CD, WD1 and WD5 males were 385 microinjected into zygotes at the Center for Transgenic Models (University of Basel, 386 Switzerland) following the same procedure as described in (4). The resulting progenies were 387 called F1-RNA-CD, F1-RNA-WD1 and F1-RNA-WD5 progenies, respectively. F2-RNA and 388 F3-RNA progenies were obtained after crossing F1-RNA and F2-RNA 4-month-old males, 
respectively, with 7-week-old C57BL/6J female mice (CD-fed) obtained from Charles River

390 (Figure 5A).

391 All mouse experiments were conducted in accordance with the National and European 392 legislations for the care and use of research animals.

393

\section{Body weight and food intake}

Body weights were measured every week from weaning until 5 months of age. Daily food consumption was estimated by weighing the remaining food every week.

For organ measurement, 5-month-old mice were anesthetized with sodium pentobarbital and rapidly dissected. Then, gonadal WAT, inguinal subcutaneous WAT, epididymis, liver and kidneys were carefully isolated, cleaned of unrelated materials and weighed. One part was fixed in 4\% PFA, whereas the other one was snap frozen in liquid nitrogen.

\section{Blood metabolic parameter measurements}

Blood metabolic parameters were detected under different physiological conditions, i.e., a random-fed state and a 16-h fasted state. Whole-blood glucose levels were determined using the OneTouch Vita (LifeScan, Johnson \& Johnson company) system from tail blood. For plasma preparation, the blood was collected from the orbital sinus into sterile $1.5-\mathrm{ml}$ tubes containing 2 drops of citrate sodium ( $3 \mathrm{M})$ and mixed gently. Blood cells were removed by centrifugation at $2000 \mathrm{xg}$ for $10 \mathrm{~min}$ at $4^{\circ} \mathrm{C}$, and the resulting supernatant was immediately aliquoted and stored at $-80^{\circ} \mathrm{C}$. Serum CRP, leptin, adiponectin and cholesterol levels were measured with the C-Reactive Protein ELISA (Mouse CRP, Elabscience, CliniSciences S.A.S., Nanterre, France), Leptin ELISA (ASSAYPRO, CliniSciences S.A.S., Nanterre, France), Adiponectin ELISA (mouse Adiponectin, EZMADP-60K, EMD Millipore Corporation, Darmsbalt, Germany) and Cholesterol Assay (Abcam, Paris, France) kits, respectively. All measurements were performed in accordance with the manufacturers' instructions.

\section{Glucose and insulin tolerance tests}


414 Mice were placed in new cages prior to starvation. For GTTs, 12-h fasted mice were injected

415 i.p. with a solution of sterile glucose ( $2 \mathrm{~g} / \mathrm{kg}$ body weight) freshly prepared in $0.9 \%$ sterile 416 saline. For ITTs, 6 -h fasted mice were injected i.p. with insulin diluted to $0.08 \mathrm{mU} / \mu \mathrm{l}$ in sterile 417 saline for a final delivery of $0.8 \mathrm{mU} / \mathrm{g}$ body weight. Baseline glucose measurements were 418 analyzed from tail blood before i.p. glucose or insulin injection ( $2 \mathrm{mg} / \mathrm{g}$ body weight) using the

419 OneTouch Vita (LifeScan, Johnson \& Johnson company) system. Blood glucose 420 measurements were taken from the tail blood at the indicated points.

421 gWAT morphometry staining

422 gWAT was fixed with Antigenfix (Microm Microtech, France), embedded in paraffin, 423 sectioned and stained with a hematoxylin and eosin solution. Slides (4/group) were scanned 424 with Axio-scan, which allowed the scanning of the entire slide at high resolution. Six pictures 425 of six different areas from 1-2 sections per sample were chosen and analyzed with image 426 analyzer software (ImageJ). Total areas of adipocytes were traced manually. The total count 427 ranged from 3275 to 7052 adipocytes per condition. The mean surface area of the 428 adipocytes was calculated using image analyzer software (ImageJ). For each sample, 4004291000 adipocytes were counted.

\section{Estimation of adipocyte number in gWAT}

431 To estimate the number of adipocytes in gWAT depots, we applied a mathematical equation 432 developed by Jo and colleagues(39), as previously described in (21). Briefly, the number of 433 adipocytes $(\mathrm{N})$ was estimated by dividing the WAT mass (M) by the density of adipocytes (D $434=915 \mathrm{~g} / \mathrm{L})$ multiplied by the mean volume of adipocytes within the WAT (V). The mean 435 volume of adipocytes is calculated from the mean diameters of adipocytes, extracted from 436 tissue sections images. The equation is presented below:

$$
N=\frac{M}{\left(D \times \frac{4}{3} \times \pi r^{3}\right)}
$$

437 Computed tomography of mice 
438 Anesthetized animals were placed in a SkyScan $\mu$ CT-1178 X-ray tomograph (Bruker) and 439 analyzed as previously described(40). Mice were scanned using the following parameters: $440104 \mu \mathrm{m}$ pixel size, $49 \mathrm{kV}, 0.5$-mm-thick aluminum filter and a rotation step of $0.9^{\circ}$. 3D 441 reconstructions and analysis of whole abdominal fat were performed using NRecon and 442 CTAn software (Skyscan), respectively, between thoracic 13 and sacral 4 vertebral markers.

\section{Liver triglyceride measurement}

444 Frozen small pieces of liver were placed in $2 \mathrm{ml}$ tubes with Ceramic Beads (for Precellys 445 homogenizer) and were homogenized in Sodium Acetate (0.2M, $\mathrm{pH} 4.5)$ using the Precellys 446 homogenizer. After centrifugation, the supernatant was stored at $-80^{\circ} \mathrm{C}$. The triglycerides in 447 homogenates were measured according to the reagent kit instruction (Triglycerides FS 448 DiaSys Diagnostic Systems GmbH, Holzheim, Germany).

\section{Histological liver examination}

Livers were prepared and fixed in $4 \%$ paraformaldehyde, embedded in paraffin, cut into 5$451 \mu \mathrm{m}$-thick slices, stained with haematoxylin and eosin (H\&E), mounted with neutral resins and 452 then scanned with Axio-scan, allowing the scanning of the entire slide at high resolution. 453 Liver histology was blindly evaluated by two independent analyses using a semiquantitative 454 scale adapted from previously validated procedures (41). To that end, images from three 455 different fields in each section were collected at $20 \times$ magnification and numbers of normal 456 hepatocytes, microvesicular and macrovesicular steatosis and degenerating hepatocytes was assessed.

\section{Sperm collection}

459 Sperm were collected from the epididymis by squeezing. The cell suspension was 460 centrifuged at $1000 \mathrm{rpm}$ for $5 \mathrm{~min}$ and the supernatant containing the spermatozoa was 461 centrifuged at $3000 \mathrm{rpm}$ for $15 \mathrm{~min}$. To reduce contamination of somatic cells, the pellet was 462 submitted to hypotonic shock by resuspension in water $(250 \mu \mathrm{l})$, followed by the addition of $46315 \mathrm{ml}$ of PBS. The suspension was finally centrifuged at $3000 \mathrm{rpm}$ for $15 \mathrm{~min}$. 
465 Total RNA from epididymal adipose tissues was extracted using TRIzol reagent (Life 466 Technologies, France) according to the manufacturer's instructions. Total RNA (0.5 $\mu \mathrm{g})$ was reverse transcribed with mouse myeloblastosis virus reverse transcriptase (Promega) under standard conditions using hexanucleotide random primers according to the manufacturer's instructions. cDNA was amplified by PCR with specific primers. Real-time PCR was performed on the Light Cycler Instrument (Roche Diagnostics) using the Platinum SYBR Green kit (Invitrogen). Specific primers for mouse leptin and 2 mouse housekeeping genes used for normalization ( $\beta$-actin and 34B4 mouse genes) were purchased from Sigma (Sigma, France). We used primers for Leptin (forward, AAC CTG GAA ATG CTC TGG CTGT; reverse, ACT CGC TGT GAA TGG CCT GAA A), 36B4F (forward, TCC AGG CTT TGG GCA TCA; reverse, CTT TAT CAG CTG CAC ATC ACT CAG A), and $\beta$-actin (forward, CTA AGG CCA ACC GTG AAA AG; reverse, CCT GCT TCA CCA CCT TCT TG).

RNA preparation and microinjection

Frozen sperm were stored at $-80^{\circ} \mathrm{C}$. RNA was then extracted by the TRIzol procedure (Invitrogen). The same preparations of sperm RNAs were used for microinjection and small RNA sequencing. RNA preparations were verified by spectrometry on an Agilent Bioanalyzer 2100 apparatus. Microinjection into fertilized eggs was performed as previously described in (26). RNA solutions were adjusted to a concentration of 1-2 $\mu \mathrm{g} / \mathrm{ml}$, and 1-2 pl were microinjected into the pronucleus of C57BL/6 fertilized mouse oocytes.

Total RNA was isolated from gonadal adipose tissue (eWAT; $n=9$ ) samples using the Ambion RiboPure (Thermo Fisher Scientific). RNA was quantified in a Nanodrop ND-1000 spectrophotometer and RNA purity and integrity was checked by using a Bioanalyzer-2100 equipment (Agilent Technologies, INC., Santa Clara, CA). Libraries were prepared using the

489 TruSeq RNA Sample Preparation Kit (llumina Inc., CA) and were paired-end sequenced (2 × 
$49075 \mathrm{bp}$ ), by using the TruSeq SBS Kit v3-HS (Illumina Inc., CA), in a HiSeq 2000 platform

491 (Illumina Inc., CA). More than 30 M PE reads were obtained for all samples.

\section{Transcriptomics analysis (RNA-sequencing analysis)}

Raw sequence files were subjected to quality control analysis using FastQC. In order to avoid low quality data, adapters were removed by Cutadapt and lower quality bases were trimmed by trimmomatic (42). The quality-checked reads processed were mapped to the mouse reference genome GRCm38/mm10 using STAR (43). Read abundance was evaluated for each gene followed by annotation versus mouse GTF by using the featureCounts function. The R package Edger was used in order to normalize the reads and identify differentially expressed (DE) genes (44). Genes with FDR $<0.05$ after correcting for multiple testing were classified as DE (45). The pheatmap and VolcanoPlot functions ( $R$ packages) were generated to graphically represent the expression levels (log2FC) and significance of DE genes among treatments. Next generation sequence data have been deposited in the GEO Database with accession number (GSE148972) and a review access token (ovwzywcgnpublor).

\section{Small RNA-sequencing analysis}

The experiment was carried out in triplicate. RNA libraries were prepared starting from 50$100 \mathrm{ng}$ of total RNA from individual mice ( $\mathrm{n}=3$ per group, 3 groups in total) and constructed using the Illumina TruSeq Stranded Small RNA Sequencing kit (Illumina) according to the manufacturer's instructions. Sequencing was performed at the Genomix platform (SophiaAntipolis, France) using the HiSeq 2500 (Illumina).

Read quality was assessed using FastQC and trimmed, against known common Illumina adapter/primer sequences, using trimmomatic. The SmallRNAs UCAGenomix pipeline with Illumina adaptor trimming was used, read sizes $<15$ nucleotides were discarded. In order to describe the general distribution of sperm small non-coding RNAs (sncRNAs), trimmed reads kept were mapped to small RNA database using a recently developed annotation pipeline, SPORTS1.0 (27). We used the default settings and database files for the mouse genome 
517 GRCm38/mm10 that are available on the Sports github

518 (https//github.com/junchaoshi/sports1.0). Averages summarized over biotypes were based 519 on the default annotation result output files. Normalization of read abundance and differential 520 expression analysis was performed by using DESeq R package on the Sports output files.

521 The baseMean for each gene, the maximum of mean counts among all conditions, was at 522 least 50 counts. NGS experiments have been deposited in the GEO Database with 523 accession number (GSE138989).

\section{Statistics and reproducibility}

525 Statistical analyses were performed using the Kruskal-Wallis test followed by the two-stage 526 step-up method of Benjamini, Krieger and Yekuteil for multiple comparisons of body weight, 527 body composition, cholesterol, Adiponectin, CRP and leptin levels, as well as leptin mRNA expression and AUC-GTT and AUC-ITT within the WD cohorts, F1-, F2-, and F3-progenies and RNA-microinjected progenies. For each parameter, all groups were compared to each

530 other in a single Kruskal-Wallis test followed by the two-stage step-up method of Benjamini,

531 Krieger and Yekuteil to adjust for all the performed multiple comparisons.

532 To determine whether the distribution of the total body weight mass was bimodal, we used 533 the Hartigans' Dip Test for unimodality/ multimodality available in the R Package 'diptest'. In 534 this dip test, if $p<0.05$, we rejected the null hypothesis of unimodality and concluded that the 535 distribution has more than one mode. Unless indicated otherwise, the unimodal distribution of 536 total body weight mass was confirmed.

537 To measure the linear relationship between two variables, we used Spearman's correlation

538 coefficient. All statistical analyses were performed with Prism 7 for Mac OS X software 539 (GraphPad software, Inc.). Data are presented as the median (IQR). $p_{a d j}<0.05$ was 540 considered statistically significant.

541 Sample size and replicates are indicated in the figure legends. The WD cohort and WD 542 progenies were repeated twice. 


\section{Acknowledgments}

545 We are grateful to Dr Jean-Jacques Remy for his careful help from the start of this project.

546 We thank Drs Mireille Cormont, Sofia Fazio, Maria Stathopoulou and Claire Mauduit for 547 constructive discussions. We thank Marion Dussot for her technical assistance in performing 548 the liver biochemistry. We relied on sequencing data generated by the IPMC Functional 549 Genomics Facility (UCAGenomiX - IPMC platform (Sophia-Antipolis, France)). We thank the 550 Center for Transgenic Models (University of Basel, Switzerland) for the mouse microinjection 551 assays. We are grateful to the C3M mouse facility (U1065, Nice). This work has been 552 supported by ANR (grant\# ANR-12-ADAPT-0022) and the FFAS "Fonds Français pour 553 l'Alimentation et la Santé "(15D52) and was partly supported by research funding from the 554 Canceropôle PACA, Institut National du Cancer and Région Sud. F.S. was supported by the UCA-IDEX.

\section{Competing Interests}

The authors declare no competing interests.

\section{References}

1. Chen Q, Yan M, Cao Z, Li X, Zhang Y, Shi J, et al. Sperm tsRNAs contribute to 560 intergenerational inheritance of an acquired metabolic disorder. Science. 561 2016;351(6271):397-400.

562 2. Fullston T, Ohlsson Teague EM, Palmer NO, DeBlasio MJ, Mitchell M, Corbett M, et 563 al. Paternal obesity initiates metabolic disturbances in two generations of mice with 564 incomplete penetrance to the F2 generation and alters the transcriptional profile of testis and sperm microRNA content. Faseb j. 2013;27(10):4226-43. 3. Grandjean V, Fourre S, De Abreu DA, Derieppe MA, Remy JJ, Rassoulzadegan M. RNA-mediated paternal heredity of diet-induced obesity and metabolic disorders. Sci Rep. 2015;5:18193. sperm RNAs in transgenerational inheritance of the effects of early trauma in mice. Nat Neurosci. 2014;17(5):667-9. Mechanisms Underlying the Non-Genomic Transmission of Obesity/Diabetes via the Maternal or the Paternal Line. Nutrients. 2019;11(2).

575 6. Remy JJ. Stable inheritance of an acquired behavior in Caenorhabditis elegans. Current biology : CB. 2010;20(20):R877-8. 
7. Skinner MK, Guerrero-Bosagna C, Haque MM. Environmentally induced

578 epigenetic transgenerational inheritance of sperm epimutations promote genetic mutations. Epigenetics. 2015;10(8):762-71.

8. Agarwal A, Majzoub A. Free Radicals in Andrology. Jannini AA, Maggi M, Foresta C, Lenzi A, editors2017.

9. Carone BR, Fauquier L, Habib N, Shea JM, Hart CE, Li R, et al. Paternally induced transgenerational environmental reprogramming of metabolic gene expression in mammals. Cell. 2010;143(7):1084-96.

10. Soubry A. POHaD: why we should study future fathers. Environmental epigenetics. 2018;4(2):dvy007.

11. de Castro Barbosa T, Ingerslev LR, Alm PS, Versteyhe S, Massart J, Rasmussen M, et al. High-fat diet reprograms the epigenome of rat spermatozoa and transgenerationally affects metabolism of the offspring. Molecular metabolism. 2016;5(3):184-97.

12. Ge ZJ, Liang QX, Hou Y, Han ZM, Schatten H, Sun QY, et al. Maternal obesity and diabetes may cause DNA methylation alteration in the spermatozoa of offspring in mice. Reprod Biol Endocrinol. 2014;12:29.

13. Ost A, Lempradl A, Casas E, Weigert M, Tiko T, Deniz M, et al. Paternal diet defines offspring chromatin state and intergenerational obesity. Cell. 2014;159(6):1352-64.

14. Terashima M, Barbour S, Ren J, Yu W, Han Y, Muegge K. Effect of high fat diet on paternal sperm histone distribution and male offspring liver gene expression. Epigenetics. 2015;10(9):861-71.

15. Sharma U, Conine CC, Shea JM, Boskovic A, Derr AG, Bing XY, et al. Biogenesis and function of tRNA fragments during sperm maturation and fertilization in mammals. Science. 2016;351(6271):391-6.

16. Cropley JE, Eaton SA, Aiken A, Young PE, Giannoulatou E, Ho JW, et al. Malelineage transmission of an acquired metabolic phenotype induced by grand-paternal obesity. Molecular metabolism. 2016;5(8):699-708.

17. Huypens $\mathrm{P}$, Sass $\mathrm{S}, \mathrm{Wu} \mathrm{M}$, Dyckhoff $\mathrm{D}$, Tschop M, Theis F, et al. Epigenetic germline inheritance of diet-induced obesity and insulin resistance. Nat Genet. 2016;48(5):497-9.

18. Massiera F, Barbry P, Guesnet P, Joly A, Luquet S, Moreilhon-Brest C, et al. A Western-like fat diet is sufficient to induce a gradual enhancement in fat mass over generations. J Lipid Res. 2010;51(8):2352-61.

19. Burcelin R, Crivelli V, Dacosta A, Roy-Tirelli A, Thorens B. Heterogeneous metabolic adaptation of C57BL/6J mice to high-fat diet. Am J Physiol Endocrinol Metab. 2002;282(4):E834-42.

20. Liberzon A. A description of the Molecular Signatures Database (MSigDB) Web site. Methods Mol Biol. 2014;1150:153-60.

21. Gilleron J, Bouget G, Ivanov S, Meziat C, Ceppo F, Vergoni B, et al. Rab4b Deficiency in $\mathrm{T}$ Cells Promotes Adipose Treg/Th17 Imbalance, Adipose Tissue Dysfunction, and Insulin Resistance. Cell Rep. 2018;25(12):3329-41.e5.

22. Lecoutre S, Oger F, Pourpe C, Butruille L, Marousez L, Dickes-Coopman A, et al. Maternal obesity programs increased leptin gene expression in rat male offspring via epigenetic modifications in a depot-specific manner. Molecular metabolism. 2017;6(8):922-30.

23. Masuyama H, Mitsui T, Eguchi T, Tamada S, Hiramatsu Y. The effects of paternal high-fat diet exposure on offspring metabolism with epigenetic changes in the mouse 
adiponectin and leptin gene promoters. Am J Physiol Endocrinol Metab. 2016;311(1):E236-45.

627 24. Schierwagen R, Maybuchen L, Zimmer S, Hittatiya K, Back C, Klein S, et al. Seven weeks of Western diet in apolipoprotein-E-deficient mice induce metabolic syndrome and non-alcoholic steatohepatitis with liver fibrosis. Sci Rep. 2015;5:12931.

25. Breyer MD, Bottinger E, Brosius FC, 3rd, Coffman TM, Harris RC, Heilig CW, et al. Mouse models of diabetic nephropathy. J Am Soc Nephrol. 2005;16(1):27-45.

26. Sarker G, Sun W, Rosenkranz D, Pelczar P, Opitz L, Efthymiou V, et al. Maternal overnutrition programs hedonic and metabolic phenotypes across generations through sperm tsRNAs. Proc Natl Acad Sci U S A. 2019;116(21):10547-56.

27. Shi J, Ko EA, Sanders KM, Chen Q, Zhou T. SPORTS1.0: A Tool for Annotating and Profiling Non-coding RNAs Optimized for rRNA- and tRNA-derived Small RNAs. Genomics Proteomics Bioinformatics. 2018;16(2):144-51.

28. Nätt D, Kugelberg U, Casas E, Nedstrand E, Zalavary S, Henriksson P, et al. Human sperm displays rapid responses to diet. PLoS Biol. 2019;17(12):e3000559.

29. Zhang Y, Zhang X, Shi J, Tuorto F, Li X, Liu Y, et al. Dnmt2 mediates intergenerational transmission of paternally acquired metabolic disorders through sperm small non-coding RNAs. Nat Cell Biol. 2018;20(5):535-40.

30. Dumas ME, Rothwell AR, Hoyles L, Aranias T, Chilloux J, Calderari S, et al. Microbial-Host Co-metabolites Are Prodromal Markers Predicting Phenotypic Heterogeneity in Behavior, Obesity, and Impaired Glucose Tolerance. Cell Rep. 2017;20(1):136-48.

31. Golay A, Ybarra J. Link between obesity and type 2 diabetes. Best practice \& research Clinical endocrinology \& metabolism. 2005;19(4):649-63.

32. Kumar R, Priyadarshi RN, Anand U. Non-alcoholic Fatty Liver Disease: Growing Burden, Adverse Outcomes and Associations. J Clin Transl Hepatol. 2020;8(1):76-86.

33. Kranz AM, Forgan LG, Cole GL, Endler JA. Light environment change induces differential expression of guppy opsins in a multi-generational evolution experiment. Evolution. 2018.

34. Jegede 00, Hale BA, Siciliano SD. Multigenerational exposure of populations of Oppia nitens to zinc under pulse and continuous exposure scenarios. Environ Toxicol Chem. 2019;38(4):896-904.

35. Horan TS, Marre A, Hassold T, Lawson C, Hunt PA. Germline and reproductive tract effects intensify in male mice with successive generations of estrogenic exposure. PLoS Genet. 2017;13(7):e1006885.

36. Hardikar AA, Satoor SN, Karandikar MS, Joglekar MV, Puranik AS, Wong W, et al. Multigenerational Undernutrition Increases Susceptibility to Obesity and Diabetes that Is Not Reversed after Dietary Recuperation. Cell Metab. 2015;22(2):312-9.

37. Fullston T, McPherson NO, Owens JA, Kang WX, Sandeman LY, Lane M. Paternal obesity induces metabolic and sperm disturbances in male offspring that are exacerbated by their exposure to an "obesogenic" diet. Physiological reports. 2015;3(3). 38. Zhou Y, Zhu H, Wu HY, Jin LY, Chen B, Pang HY, et al. Diet-Induced Paternal Obesity Impairs Cognitive Function in Offspring by Mediating Epigenetic Modifications in Spermatozoa. Obesity (Silver Spring). 2018;26(11):1749-57.

39. Jo J, Gavrilova O, Pack S, Jou W, Mullen S, Sumner AE, et al. Hypertrophy and/or Hyperplasia: Dynamics of Adipose Tissue Growth. PLoS Comput Biol. 2009;5(3):e1000324. 
672 40. Beranger GE, Pisani DF, Castel J, Djedaini M, Battaglia S, Amiaud J, et al. Oxytocin 673 reverses ovariectomy-induced osteopenia and body fat gain. Endocrinology. $6742014 ; 155(4): 1340-52$.

675 41. Kleiner DE, Brunt EM, Van Natta M, Behling C, Contos MJ, Cummings OW, et al. 676 Design and validation of a histological scoring system for nonalcoholic fatty liver 677 disease. Hepatology. 2005;41(6):1313-21.

678 42. Bolger AM, Lohse M, Usadel B. Trimmomatic: a flexible trimmer for Illumina 679 sequence data. Bioinformatics. 2014;30(15):2114-20.

680 43. Dobin A, Davis CA, Schlesinger F, Drenkow J, Zaleski C, Jha S, et al. STAR: ultrafast 681 universal RNA-seq aligner. Bioinformatics. 2013;29(1):15-21.

682 44. McCarthy DJ, Chen Y, Smyth GK. Differential expression analysis of multifactor 683 RNA-Seq experiments with respect to biological variation. Nucleic Acids Res. 684 2012;40(10):4288-97.

685 45. Love MI, Huber W, Anders S. Moderated estimation of fold change and dispersion 686 for RNA-seq data with DESeq2. Genome Biol. 2014;15(12):550.

\section{Figure Legends}

689 Figure 1. Five consecutive paternal generations of WD feeding exacerbate the WD690 induced overweight phenotype.

691 (A) Study design for the maintenance of WD feeding for 5 consecutive generations through

692 the paternal lineage. Male mice were randomized to receive either a control diet (CD; $5 \%$ of 693 energy from fat) or a western diet (WD1; 45\% of energy from fat). After 3 months of WD694 feeding, 4 males obtained from different fathers were arbitrary chosen to generate 4 695 independent families. They were mated with CD-fed females to generate WD2 offspring. At 3 696 weeks old, they were fed a WD and at 4 months, at least one WD-fed male per family were crossed with CD-fed females. This second generation of males was called WD2. This experimental design was repeated 3 times to obtain the WD5 group (Figure 1 -figure

699 supplement 1). (B) Box-whiskers (min-max) of the median total body weight of the different 700 male WD cohorts ( $\mathrm{n} \geq 8$ mice per group). (C) Box-whiskers (min-max) of the median 701 perigonadal white adipose tissue (gWAT) weight relative to total body weight in the different 702 WD cohorts. (D) H\&E staining of gWAT sections (scale bar: $200 \mu \mathrm{m}$ ) in representative CD, 703 WD1, WD4 and WD5 males. ( $E$ ) Box-whiskers (min-max) of the median surface area $\left(\mu m^{2}\right)$ 704 of the adipocytes, which was calculated using Image Analyzer software (ImageJ). The total 705 count ranged from 3275 to 7052 cells per condition ( $\mathrm{n} \geq 4$ mice per group). ( $F$ ) Box-whiskers 
706 (min-max) of the number of adipocytes which was estimated using the mathematical

707 equation developed by Jo et al.(39), as previously described in (21). (G) Table showing the

708 differentially expressed genes (DEGs) in WD1 and WD5 perigonadal white adipose tissue.

$709{ }^{*} p_{a d j}<0.05,{ }^{* *} p_{a d j}<0.01,{ }^{* * *} p_{a d j}<0.001,{ }^{* * *} p_{a d j}<0.0001$

710 Figure 2. Five consecutive paternal generations of WD feeding exacerbate WD711 induced overweight pathologies.

$712(A-B)$ Evolution of glucose parameters in male mice fed a WD for five successive

713 generations. Blood glucose and insulin tolerance tests were performed on 16-week-old

714 males $(n \geq 6)$. Plasma glucose [inserted box-whiskers (min-max) of the median area under the

715 curve (AUC) and above baseline for glucose from time point 0 to 120; glucose tolerance test]

716 (A) [inserted box-whiskers (min-max) of the median AUC and above baseline for glucose

717 from time point 0 to 100 ; insulin tolerance test] $(B)$. Glucose tolerance and insulin tolerance

718 tests were conducted in the morning in overnight-fasted mice. (C) Box-whiskers (min-max) of

719 the median liver weight relative to total body weight in the different WD cohorts $(n \geq 8$ mice per

720 group). (D) Liver triglyceride contents in the CD, WD1 and WD5 groups $(\mathrm{n} \geq 6)$. $(E)$

721 Percentage of normal hepatocytes, hepatocytes with microvesicular steatosis, hepatocytes

722 with macrovesicular steatosis and ballooning degenerative hepatocytes in CD, WD1 and

723 WD5 livers $(\mathrm{n} \geq 6)$. ( $F$ ) H\&E staining of liver sections (scale bar: $250 \mu \mathrm{m}$ ) from representative

724 CD, WD1 and WD5 males.

$725{ }^{*} p_{\text {adj }}<0.05,{ }^{* *} p_{\text {adj }}<0.01,{ }^{* * *} p_{\text {adj }}<0.001$

726 Figure 3. Maintenance of the overweight phenotype after 4 generations on the CD in 727 the progenies generated from WD5-fed males.

728 (A) Study design for the inheritance of WD-induced metabolic alterations in WD1- and

729 WD5-fed animals. Four WD1 and nine WD5 male mice from different litters (arbitrary

730 selected from the four different families) were mated with CD-fed females to generate F1-

731 WD1 and F1-WD5 offspring, respectively. Each offspring was fed the CD. This crossing

732 scheme was repeated three times to obtain the F2-, F3-, F4-WD1 and F2-, F3-, F4-WD5 
733 offspring. The number of mice is indicated. Box-whiskers (min-max) of the median total body

734 weights of 16 -week-old males $(B, D)$ and females $(F, H)$ of progenies from WD-fed animals.

735 Box-whiskers (min-max) of the median gWAT of males $(C, E)$ and females $(G, I)$ of progenies 736 from WD-fed animals.

737 The unimodality / multimodality of distributions for body weight for all groups was tested 738 using the Hartigans' Dip Test for unimodality/ multimodality. All groups, except the F1-WD1 739 male progenies, followed a unimodal distribution.

740 Gray rectangles and circles represent the male and female progenies, respectively, from 741 WD1-fed animals. Blue rectangles and red circles represent the male and female progenies, 742 respectively, from WD5-fed animals.

$743 \quad{ }^{*} p_{a d j}<0.05,{ }^{* *} p_{a d j}<0.01,{ }^{* * *} p_{a d j}<0.001$

\section{Figure 4. Sperm sncRNAs signature modulated by the ancestors' diet.}

745 (A) Representative bioanalyzer profiles of CD, WD1 and WD5 sperm total RNAs. (B) 746 Length distribution and pattern changes of sperm sncRNA different populations (miRNAs 747 piRNAs, tsRNAs and rsRNA) in one representative CD, WD1 and WD5. Each graph was 748 generated by SPORTS1.0 (27). (C) Small RNA-seq profiles of each CD, WD1 and WD5 749 male. (D) Mean proportion of each small RNA population across each group. (E) The 750 normalized small RNA levels from the CD (blue spots), WD1 (red spots) and WD5 (green 751 spots) sperm were analyzed by PCA. One WD5 fell outside the PCA cluster and was 752 arbitrarily removed for differential expression analysis. $(E)$ Venn diagram of small RNA 753 sequences differentially expressed in WD1 and WD5 sperm. The numbers of small RNAs 754 that are unique for each WD1 and WD5 male are shown in each circle. The numbers of 755 genes in overlapping (common) are indicated at the intersections of the sets in the Venn 756 diagram $\left(\mathrm{P}_{\mathrm{adj}}<0.05\right.$ Log2FC $\left.\geq|0.6|\right)$. ( $F$ ) Scatter plots of microRNAs or piRNAs, tRNA 757 fragments, and other small RNAs differentially expressed $\left(P_{\text {adj }}<0.05 \mid\right.$ Log2FC $\left.\mid \geq 0.6\right)$ in WD1 758 (left panel) and WD5 (right panel) sperm compared to their expression in the CD sperm 759 cohort. 
Figure 5. Zygotic microinjection of sperm total RNA from either WD1 or WD5 males induces metabolic alterations in the F1 and F2 CD-fed progenies that are not maintained in the F3 and F4 CD-fed progenies.

(A) Study design for the inheritance of metabolic alterations induced after the microinjection of sperm total RNA from CD-, WD1- or WD5-fed males into C57BL/6J zygotes. Five F1 CDfed males from each set of RNA microinjections were mated with CD-fed females to generate F2-RNA offspring. Each offspring was fed a control diet. This crossing scheme was repeated twice to obtain the F3-RNA offspring and then the F4-RNA offspring. $(B)$ Box-whiskers (min$\max$ ) of the median total body weight of the F1-, F2-, F3-, and F4-RNA male progenies ( $\mathrm{n} \geq 8$ mice per group). (C) Box-whiskers (min-max) of the median gWAT weight relative to total body weight in the different RNA progenies. The evolution of glucose parameters in male

772 mice from RNA-injected progenies. (D) Box-whiskers (min-max) of the median AUC-GTT of 773 each cohort. $(E)$ Box-whiskers (min-max) of the median AUC-ITT of each group. $(F)$ Bivariate

774 correlation between the body weight of the F2-RNA-CD, F2-RNA-WD1 and F2-RNA-WD5 775 progenies and the AUC-GTT $(n=38)$. This correlation was similar using parametric 776 (Pearson, $r=0.4, p=0.01$ ) or nonparametric (Spearman, $r=0.4, p=0.01$ ) correlations. ${ }^{*} p_{a d j}<0.05,{ }^{* *} p_{a d j}<0.01,{ }^{* * *} p_{a d j}<0.001$

Table 1. Evolution of serum biomarker parameters in different WD groups

\begin{tabular}{|c|c|c|c|c|c|c|}
\hline Parameters & $\begin{array}{l}\text { Control } \\
n=6\end{array}$ & $\begin{array}{l}\text { WD1 } \\
n=4\end{array}$ & $\begin{array}{c}\text { WD2 } \\
n=5\end{array}$ & $\begin{array}{l}\text { WD3 } \\
n=7\end{array}$ & $\begin{array}{l}\text { WD4 } \\
n=7\end{array}$ & $\begin{array}{l}\text { WD5 } \\
\mathrm{n}=7\end{array}$ \\
\hline Adiponectin (ng/ml) & $4.4(4.9-5.2)$ & $5.2(3.3-6.9)$ & $3.5(2.8-4.7)$ & $3.4(2.8-5.5)$ & $3.6(2.9-4.2)$ & $4.6(3.5-5.0)$ \\
\hline Leptin (ng/ml) & $6.7(5.1-7.5)$ & $10.2(6.2-11)$ & $\begin{array}{l}8.2(6.2- \\
11.6)\end{array}$ & $11.5(7.9-28)$ & $19(13-32)^{\star \star 2}$ & $21(12-26)^{\star \star 2}$ \\
\hline CRP (g/ml) & $4.2(3.2-5.0)$ & $5.8(5.1-6.2)$ & $5.7(5.4-6.2)$ & $7.1(5.7-9.8)^{\star \star}$ & $5.7(3.5-7.9)^{\star}$ & $5.8(4.6-7.4)^{\star \star}$ \\
\hline $\begin{array}{l}\text { Total cholesterol } \\
(\mathrm{mg} / \mathrm{dl})\end{array}$ & $1.1(0.9-1.2)$ & $1.8(1.3-2.0)^{\star \star}$ & $1.4(1.0-1.6)$ & $1.6(1.5-1.9)^{\star}$ & $1.9(1.6-2.4)^{\star \star \star 2}$ & $1.9(1.6-2.4)^{\star \star \star 2}$ \\
\hline
\end{tabular}


782 Values are expressed as median(IQR). Numbers are in bold if $\mathrm{p}_{\mathrm{adj}}<0.05$. ${ }^{*}$ and 2 denoted the

783 WD groups significantly different from that of the CD and WD2 group, respectively. $784{ }^{*} p_{a d j}<0.05,{ }^{* *} p_{a d j}<0.01$ 
786 Figure 1-figure supplement 1. Schematic representation of the experimental procedure.

(A) Each square corresponds to one mouse. $\mathrm{n}$ corresponds to the number of offspring for each WD group. Because of the heterogeneity of the phenotype inside the same litter from the WD2 generations, more than one progenitor from the same litter were used to obtain the

(B) Each square corresponds to one mouse. $\mathrm{n}$ corresponds to the number of offspring for each CD group. Because of the homogeneity of the phenotype inside the same litter (Figure 1, figure supplement 2D), one progenitor per litter was used to obtain the CD2, CD3 and CD4. All CD mice were mated with a CD-fed female. (C) Scatter plot of total body weight mass for each litter of all WD generations. Each data point represents the measurements of one male.

Figure 1-figure supplement 2. Exacerbation of the overweight phenotype upon continuous paternal WD feeding for multiple generations.

Evolution of total body weight at 12 and 18 weeks over WD-fed generations $(n \geq 15)$. (B-E) correlation analysis contains WD1-WD5 males $(B)$ Scatter plot of all WD litters. To

801 demonstrate that we didn't create significance with increasing the correlated sample, 802 we selected a subset of 18 mice in WD4 and WD5 making sure that the 4 WD1 ancestors were equitably represented across the groups. Three combinations of 18 mice were selected and gave the same significance level with a $p<0.001$ when total body weight mass was compared to the corresponding weight of CD group. This

806 scatter plot represents one of these combinations. (C) Box-whiskers (min-max) of the 807 median total body weights of the different male CD-fed and WD-fed cohorts. Statistical 808 significance ( $p$ value) of differences in total body weight mass between CD-fed and WD-fed 809 males from each generation were determined with the Mann-Whitney $U$ test for unpaired 810 observations (two-tailed), ${ }^{*} \mathrm{p}<0.05 ;{ }^{* *} \mathrm{p}<0.01 ;{ }^{* *} \mathrm{p}<0.001$. (D) Box-whiskers (min-max) of 811 the median total body weights of the CD-fed males in each generation. ns= not significant. 
812 (E) Positive linear correlation between gWAT and total body weight. Statistically significant

813 positive linear correlation between gWAT and plasma leptin concentration $(F)$, between

814 gWAT and plasma total cholesterol $(G)$, between gWAT and plasma CRP concentration $(H)$ 815 and between plasma leptin concentration and gWAT leptin mRNA $(I)$. (D-H) Each data point 816 represents the measurements of one male from generation 1 (WD1) to generation 5 (WD5).

817 ( $(J)$ There was no statistically significant linear correlation between epididymal fat mass and 818 AUC-GTT. (K) Principal component analysis (PCA) analysis of fasting blood glucose, total 819 body weight, epididymal fat mass and kCal in the different WD cohorts visualizing the pattern 820 of WD males depending on the number of WD-fed ancestors.

821 Figure 3-figure supplement 1. Long-term epigenetic inheritance of a "healthy" overweight phenotype in CD-fed progenies from WD5 males.

823 Box-whiskers (min-max) of the median total body weight of the male $(A)$ and female $(C) \mathrm{F} 1$-,

824 F2-, F3-WD progenies ( $\mathrm{n} \geq 8$ mice per group). Box-whiskers (min-max) of the median gonadal 825 fat mass (gWAT) weight relative to total body weight in the male $(B)$ and female $(D)$ F1-, F2-, 826 F3-WD progenies ( $n \geq 8$ mice per group). The evolution of glucose parameters in CD-fed male $827(\mathrm{E}, \mathrm{F})$ and female $(\mathrm{G}, \mathrm{H})$ WD progenies. Above the glucose tolerance curves are representative corresponding box-whiskers (min-max) of the median AUC-GTT of each group $(E, G)$. Above the insulin tolerance curves are representative corresponding box-

830 whiskers (min-max) of the median AUC-ITT of each cohort $(F, H)$ measured in each WD 831 cohort.

$832 * p<0.05 ;{ }^{* *} p<0.01 ;{ }^{* *} p<0.001$

833 Figure 3-figure supplement 2. No liver alteration was observed in the CD-fed progenies 834 from WD1 and WD5 males.

835 Liver triglyceride contents in CD, WD1 and WD5 CD-fed progenies, $n \geq 4 /$ group. (B) H\&E 836 staining of liver sections (scale bar: $250 \mu \mathrm{m}$ ) in representative F1, F2, and F3 CD-fed 837 progenies of CD, WD1 and WD5 males.

838 Figure 5-figure supplement 1. Sperm RNA microinjection did not fully recapitulate the 839 fatty liver phenotype of the WD5 father 
840 (A) Liver triglyceride contents in F1, F2, and F3 progenies of CD-RNA-, WD1-RNA- and 841 WD5-RNA-injected groups. (B) H\&E staining of liver sections (scale bar: $250 \mu \mathrm{m}$ ) in 842 representative F1, F2 and F3 CD-fed progenies of CD-RNA-, WD1-RNA- and WD5-RNA843 injected groups.

844 
A

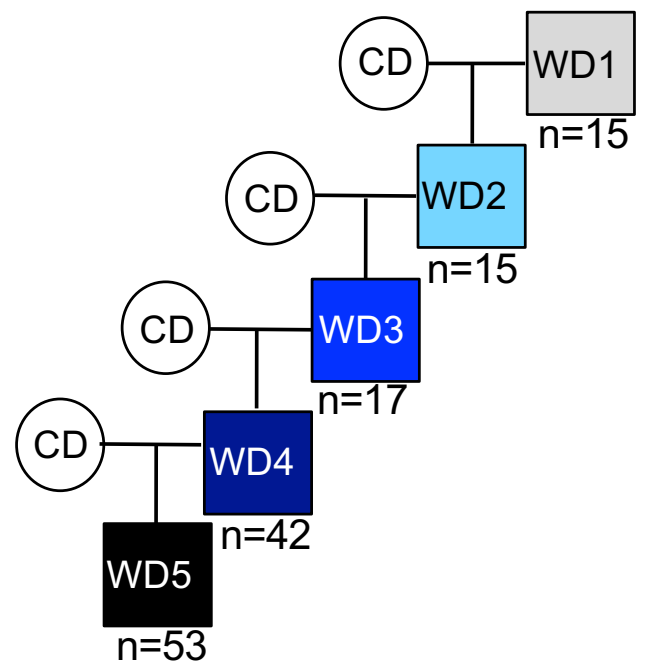

C

更

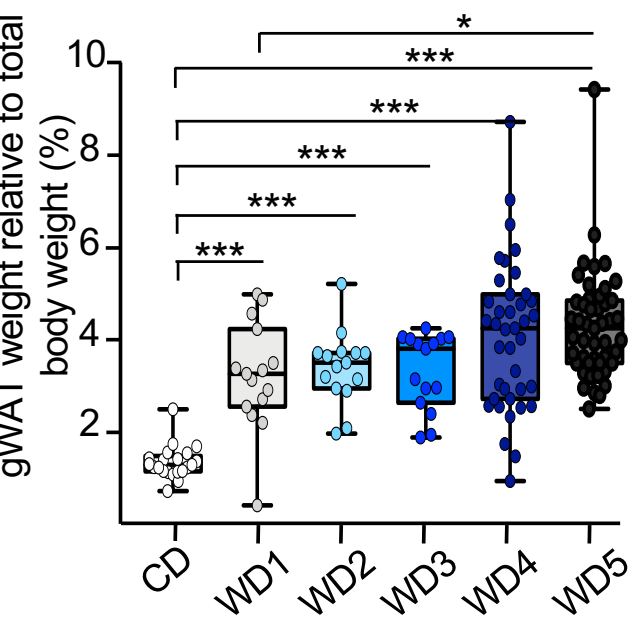

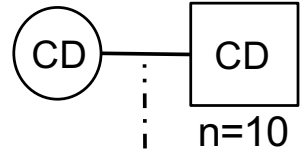

CD

WD4
B

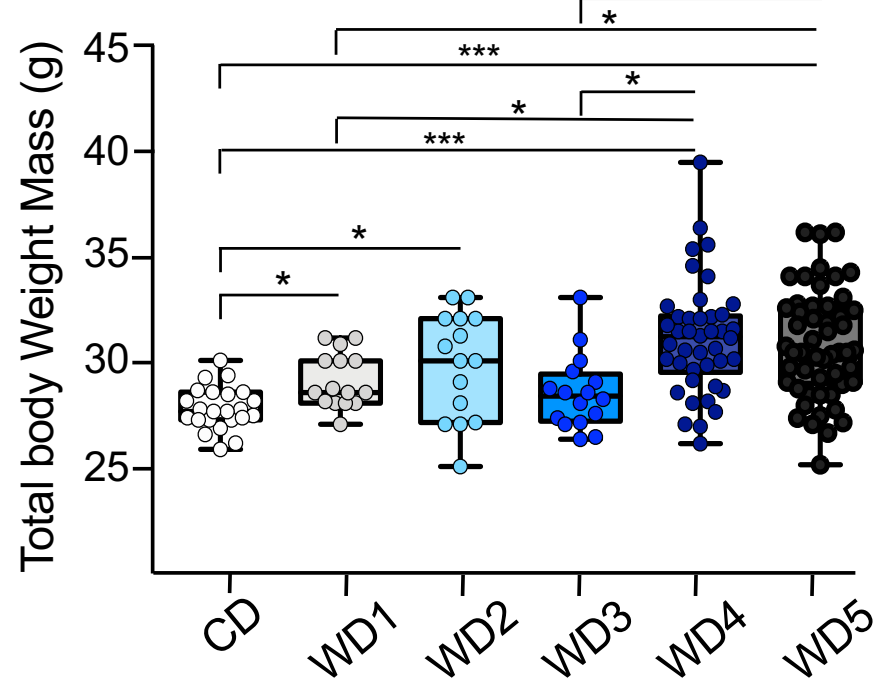

E

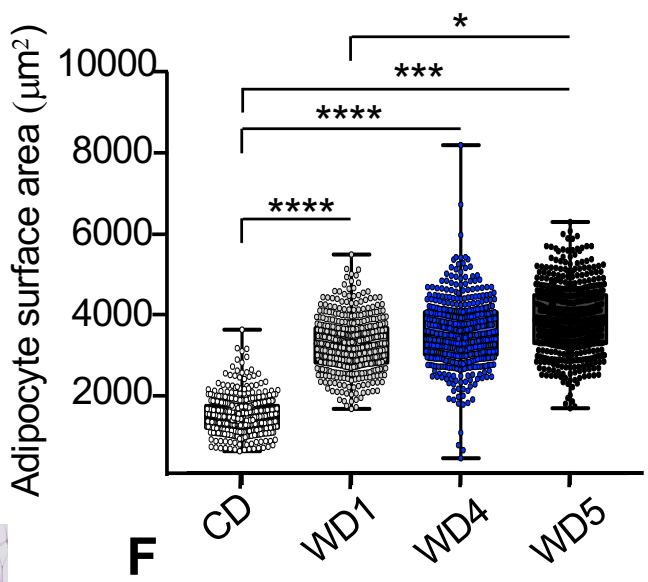

WD5

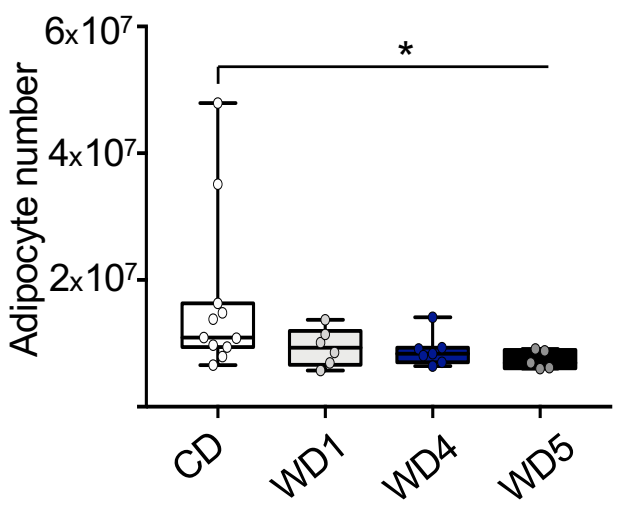

G

\begin{tabular}{|l|c|c|c|}
\hline & Total & WDs specific (\%) & Common genes (\%) \\
\hline Genes up in WD1 & 93 & $31(33)$ & $62(67)$ \\
\hline Genes down in WD1 & 232 & $79(34)$ & $153(66)$ \\
\hline Genes up in WD5 & 757 & $695(92)$ & $62(8)$ \\
\hline Genes down in WD5 & 442 & $289(65)$ & $153(35)$ \\
\hline
\end{tabular}

Figure 1 

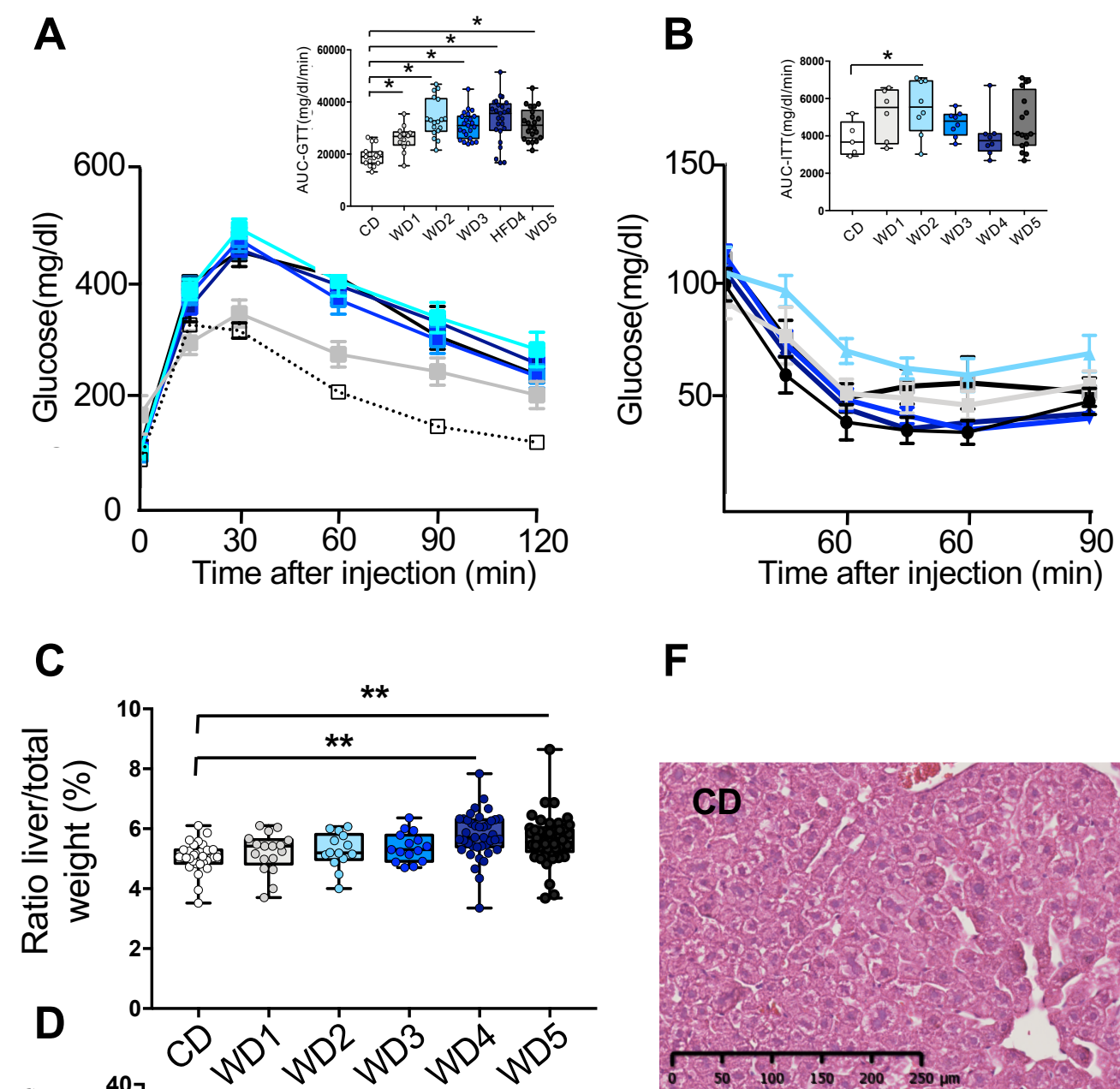

F
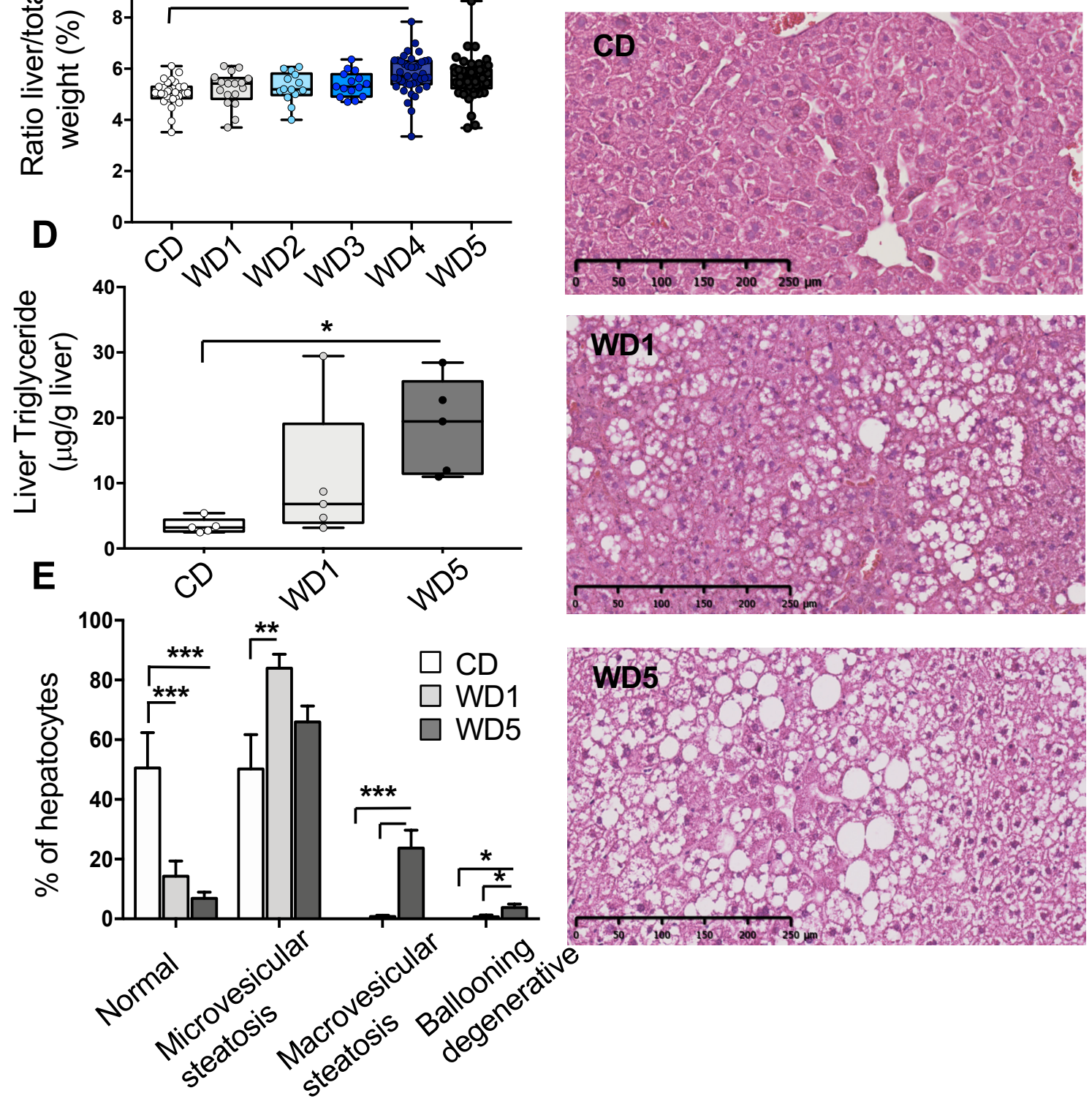

Figure 2 
A

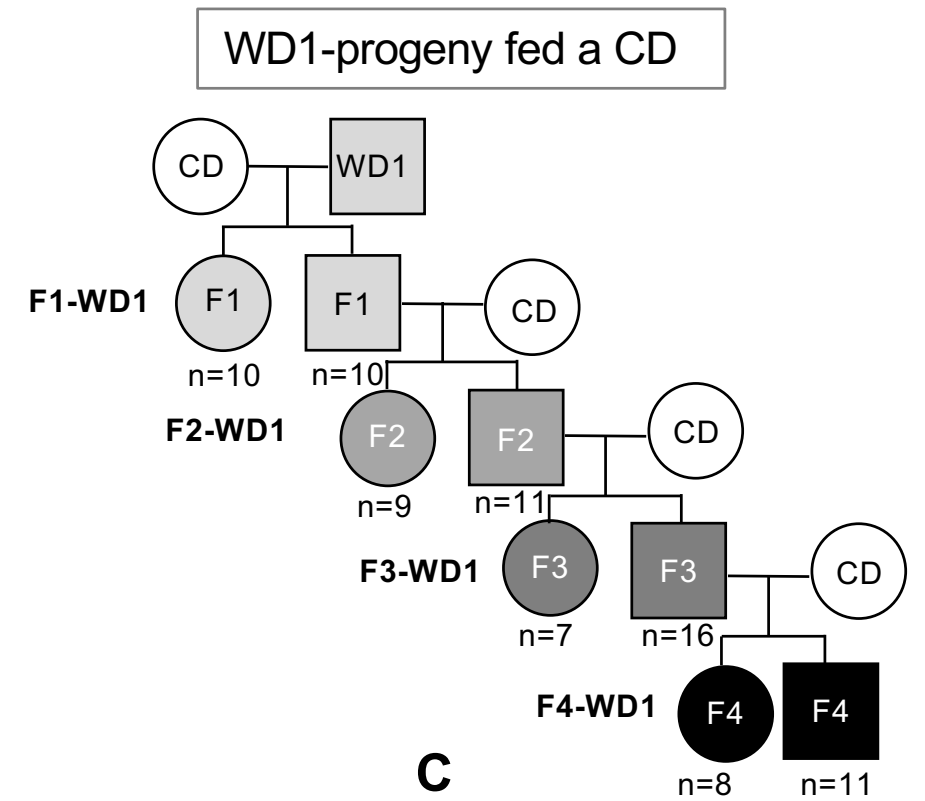

B

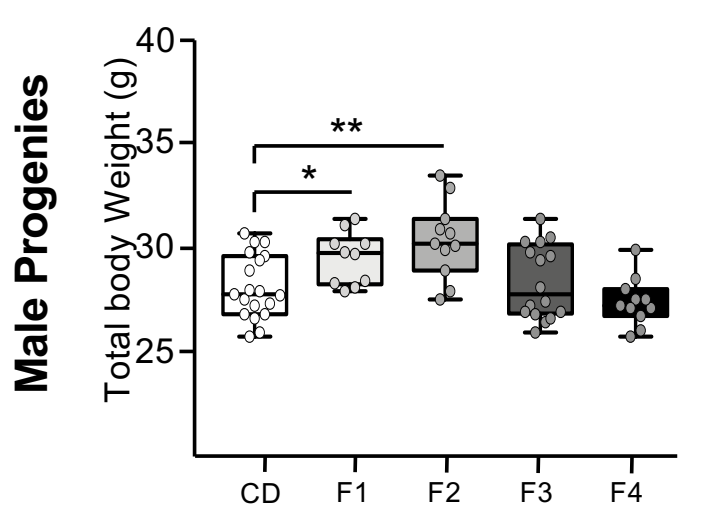

$\mathbf{F}$

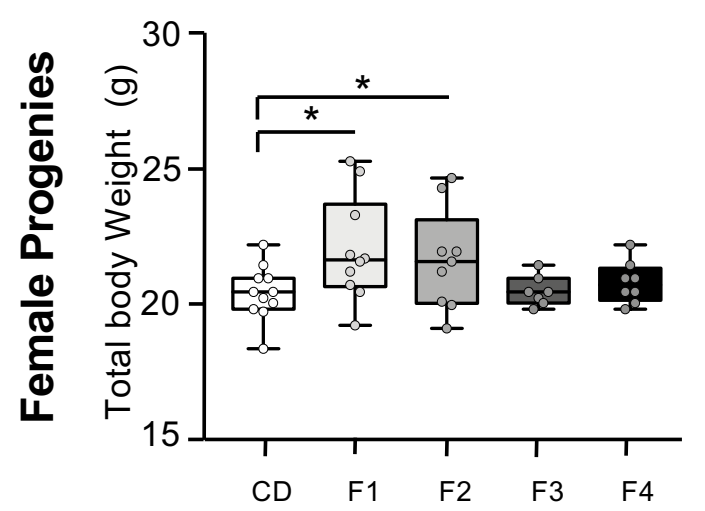

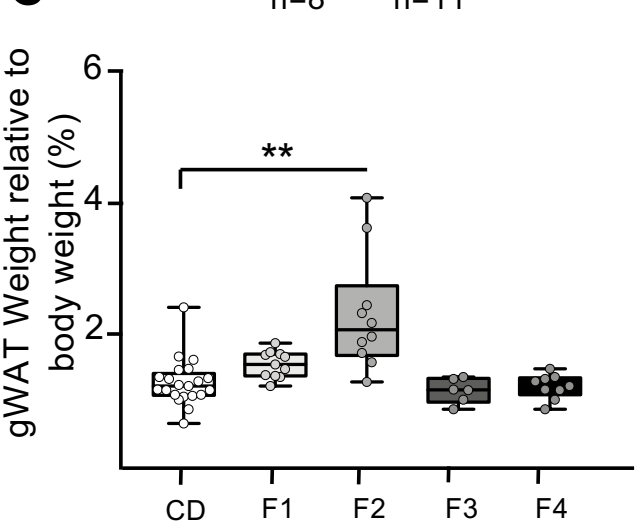

G

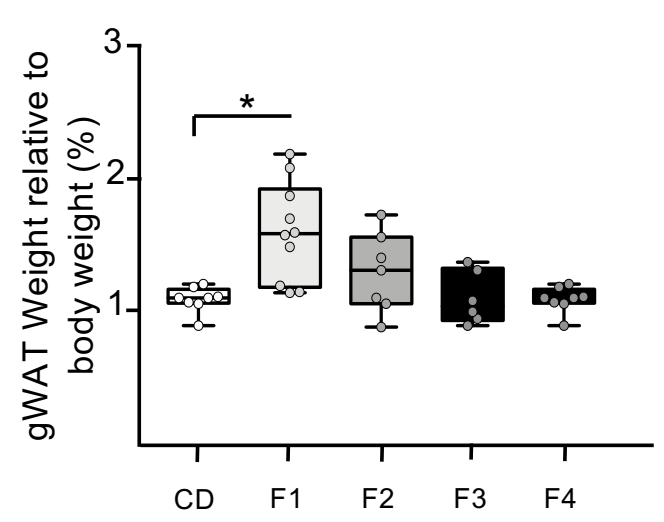

\section{WD5-progeny fed a CD}
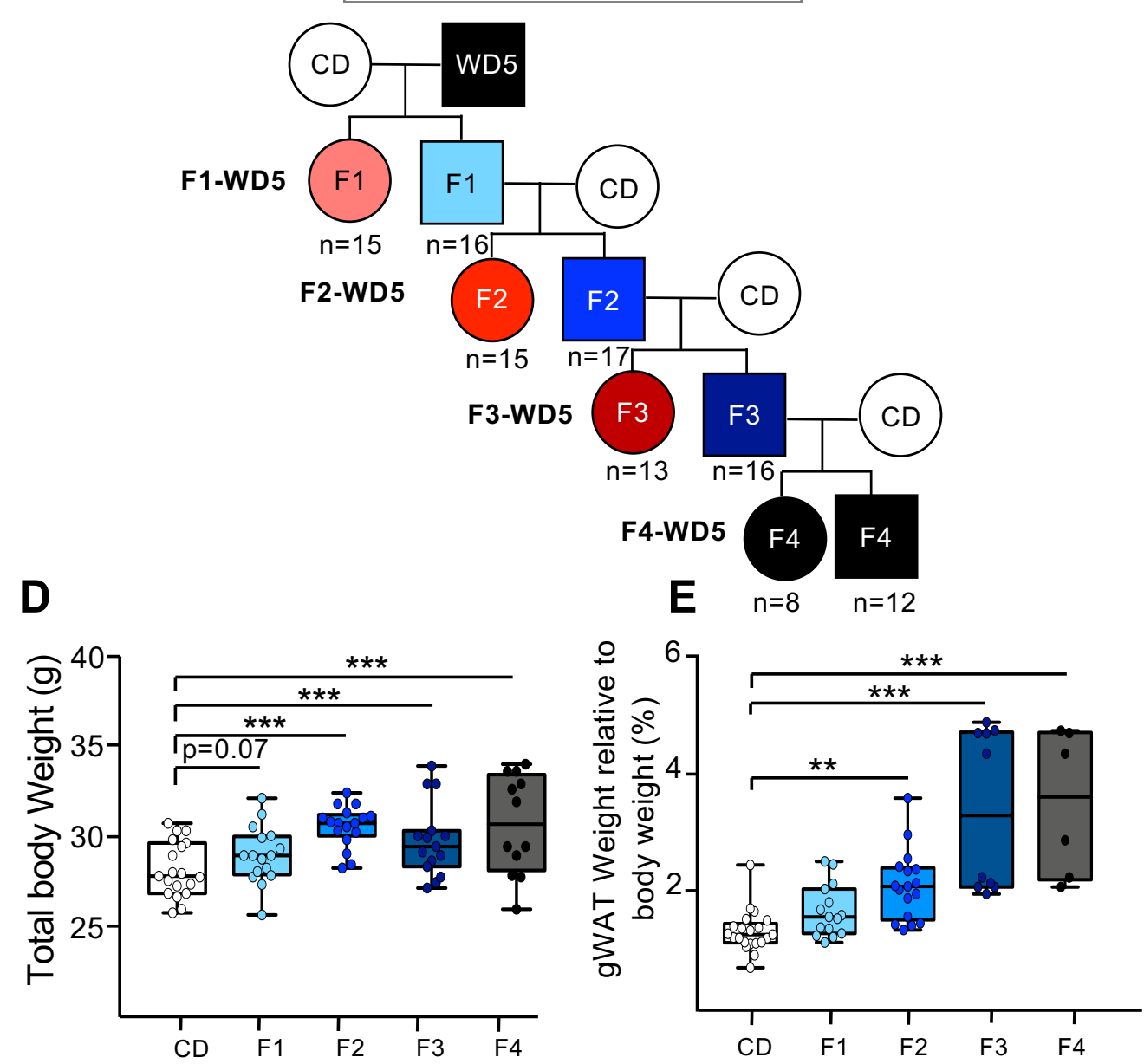

H

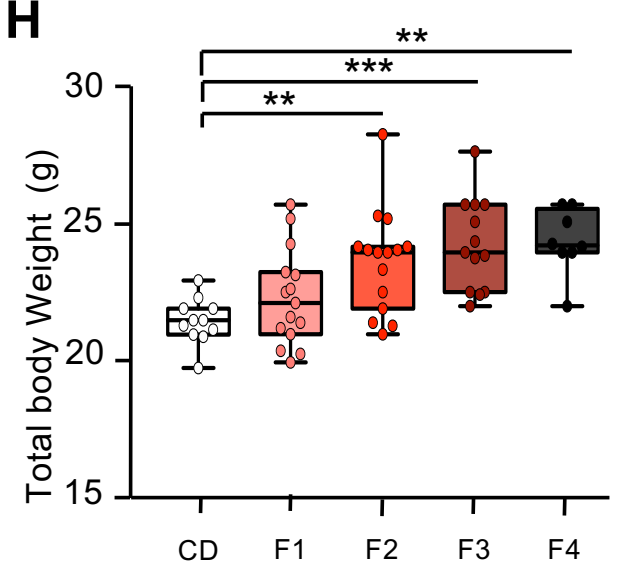

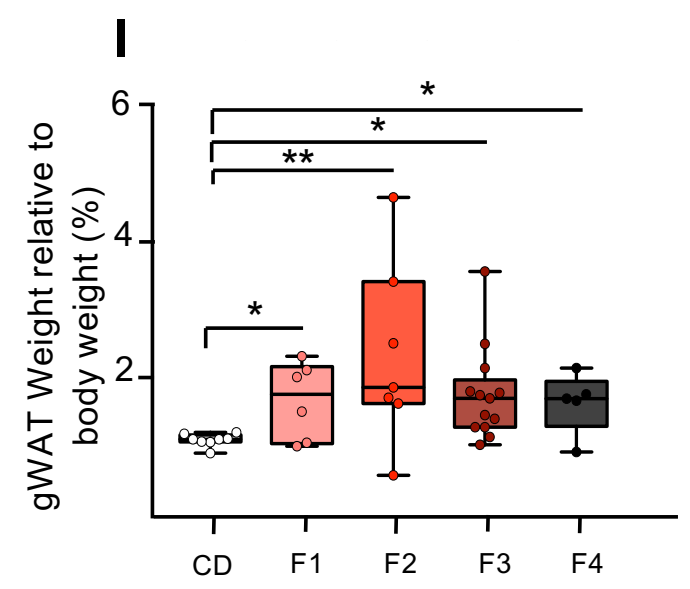


A
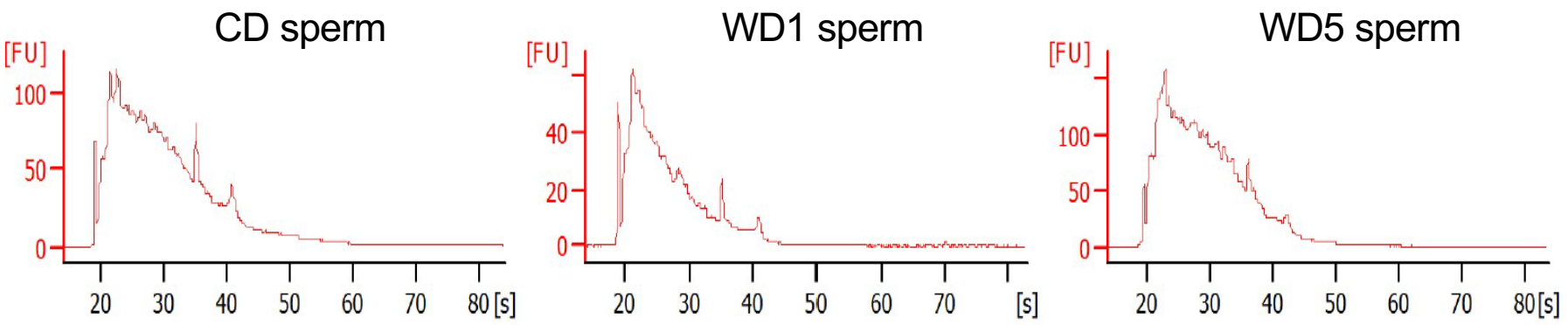

B sm Small RNAs Length Distribution of CD

Sr Small RNAs Length Distribution of WD1

Small RNAs Length Distribution of WD5
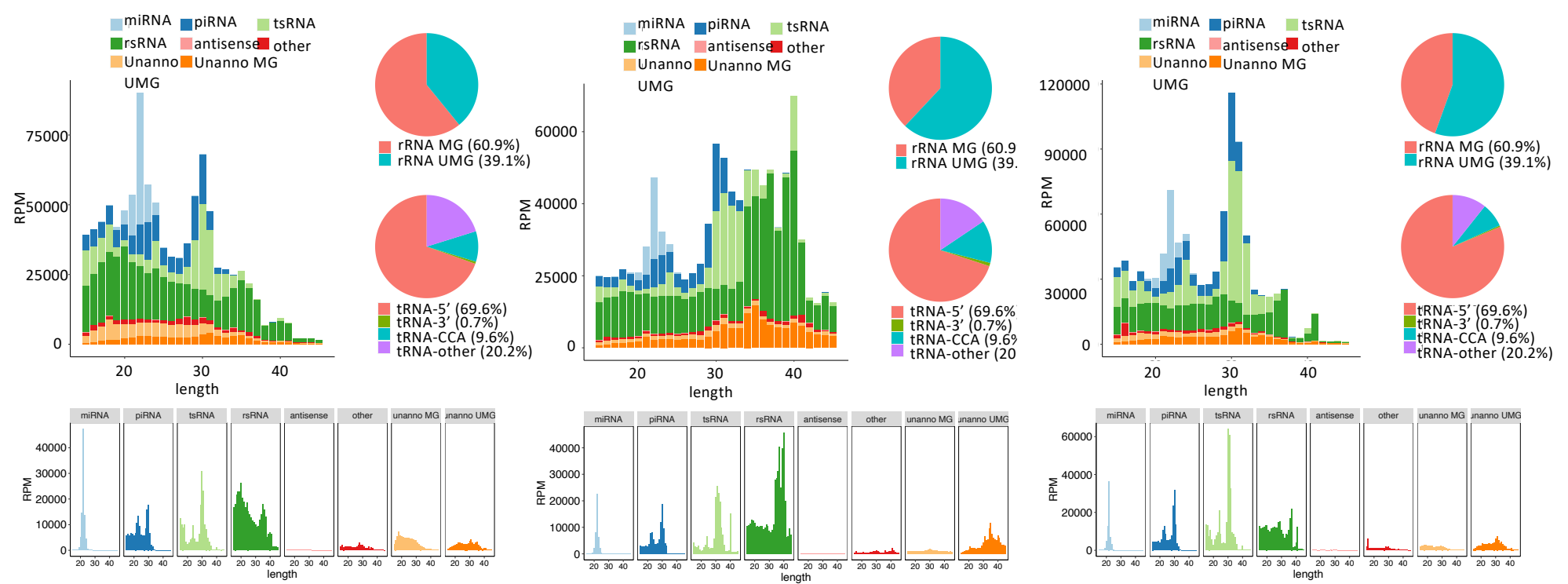

C

CD

WD1

WD5

E
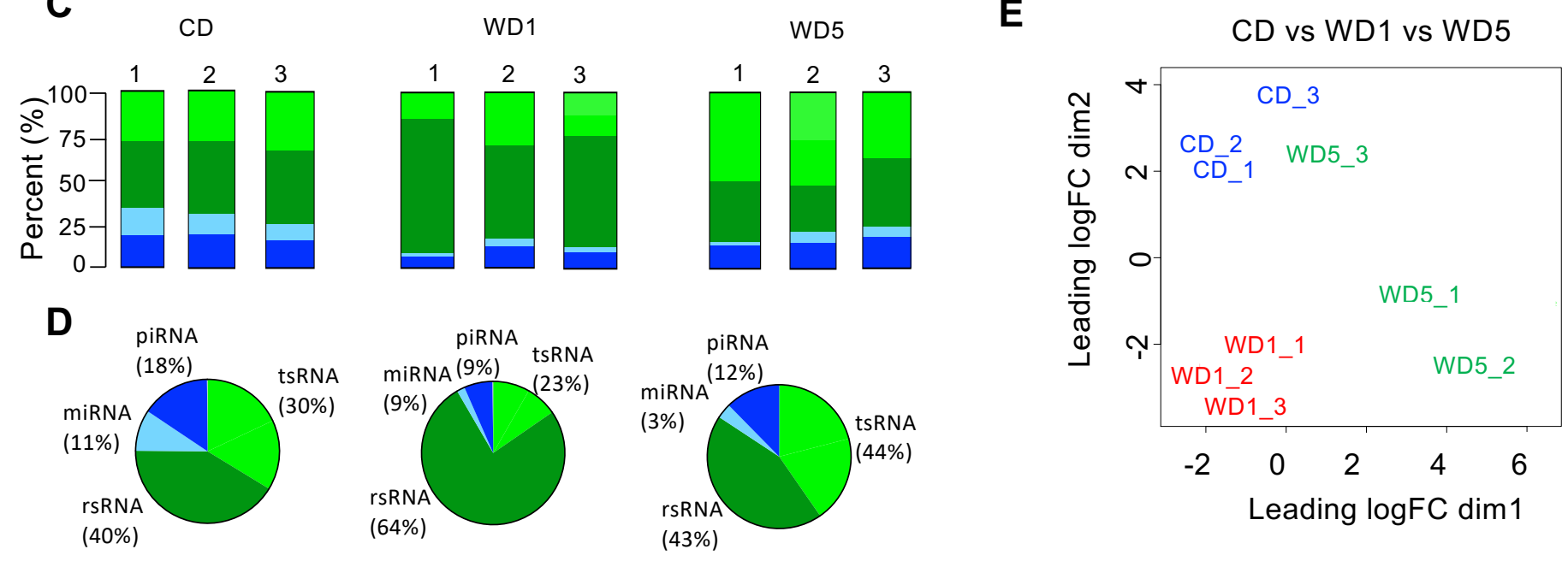

E

WD1

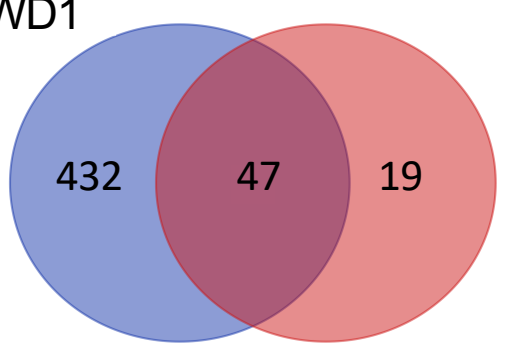

F

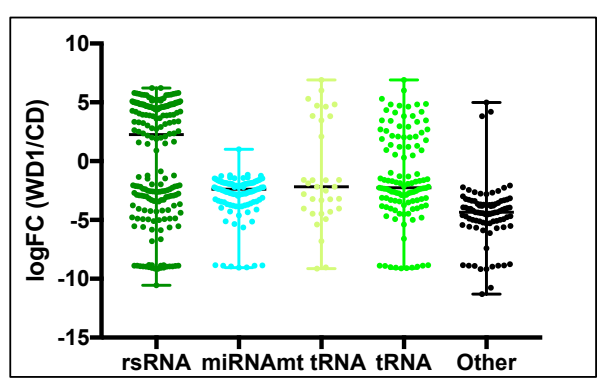

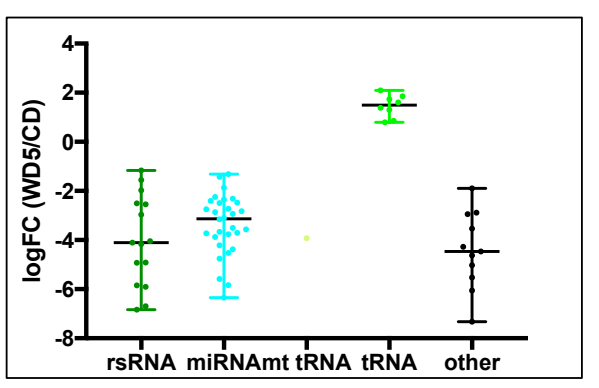

WD5 


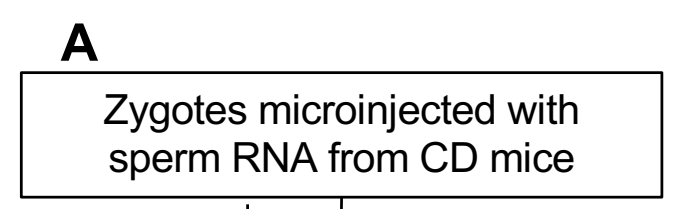

Zygotes microinjected with
sperm RNA from WD1 mice

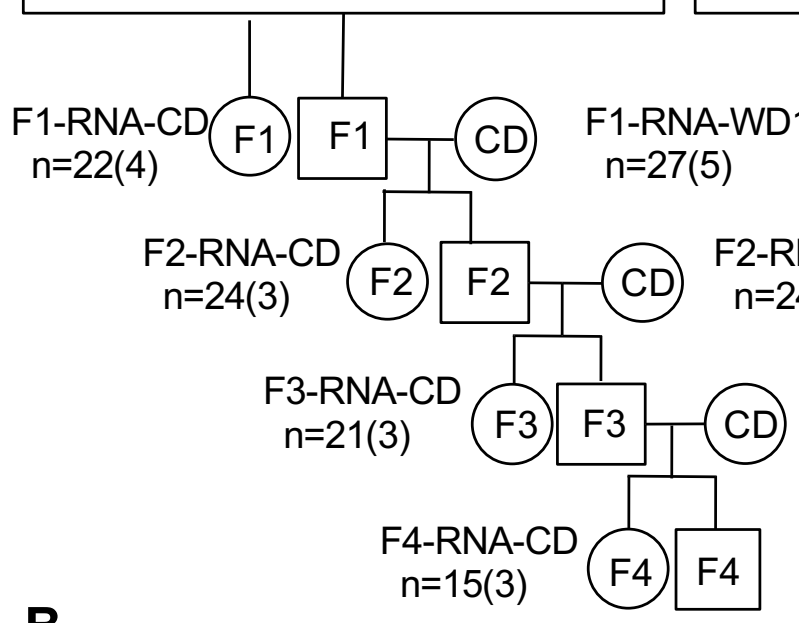

B

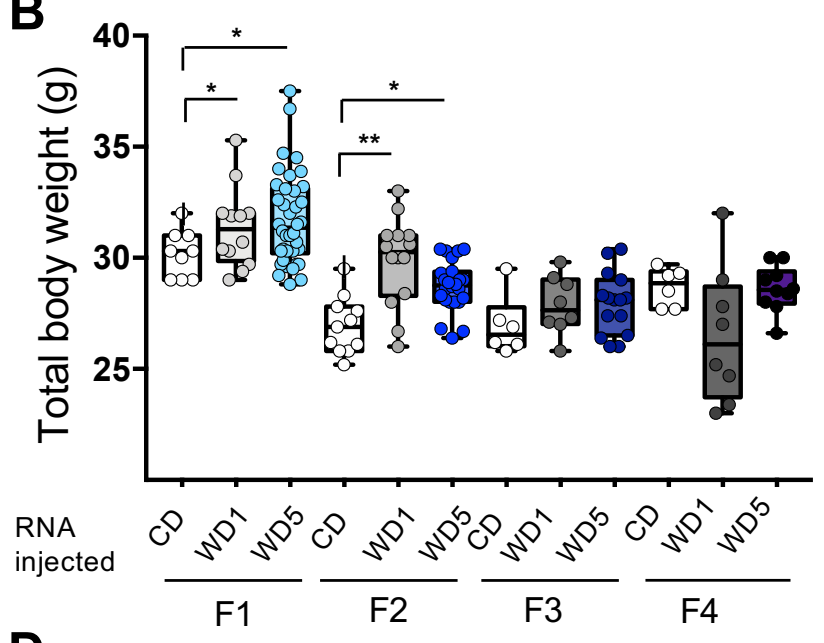

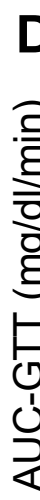
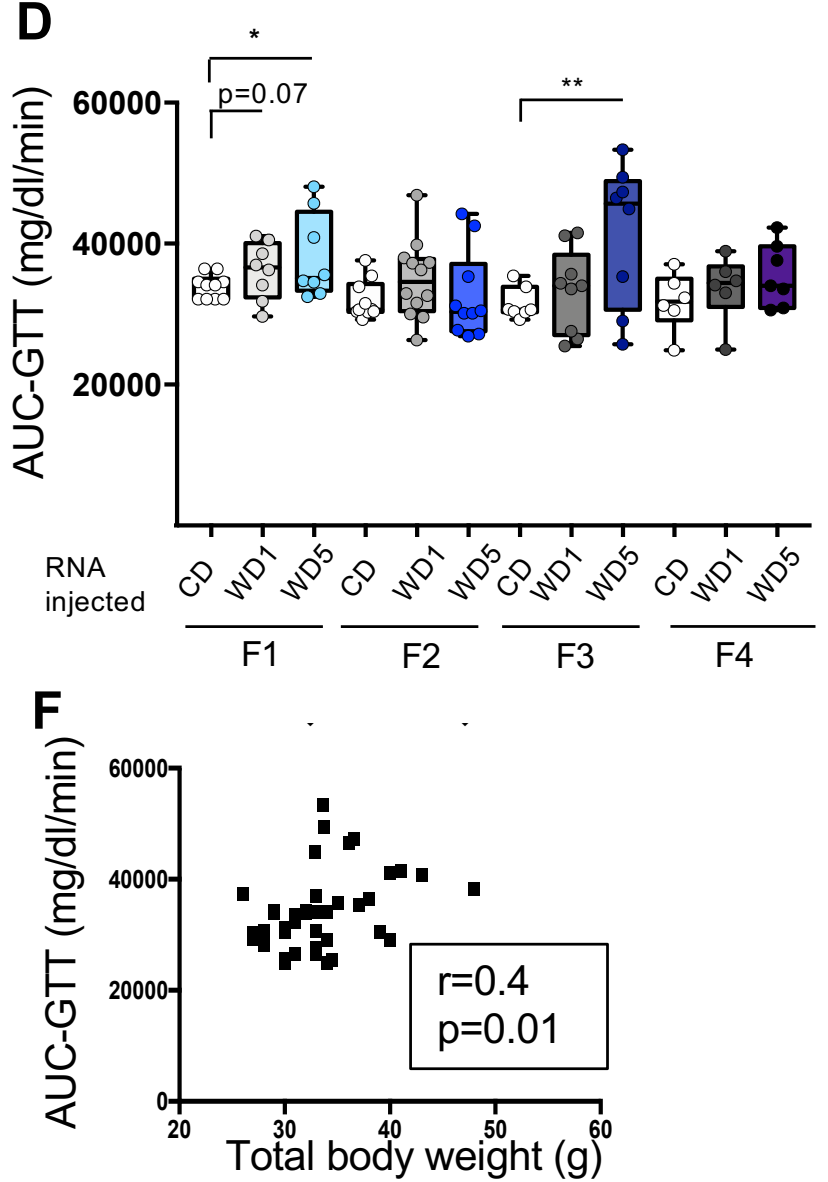

Zygotes microinjected with sperm RNA from WD5 mice

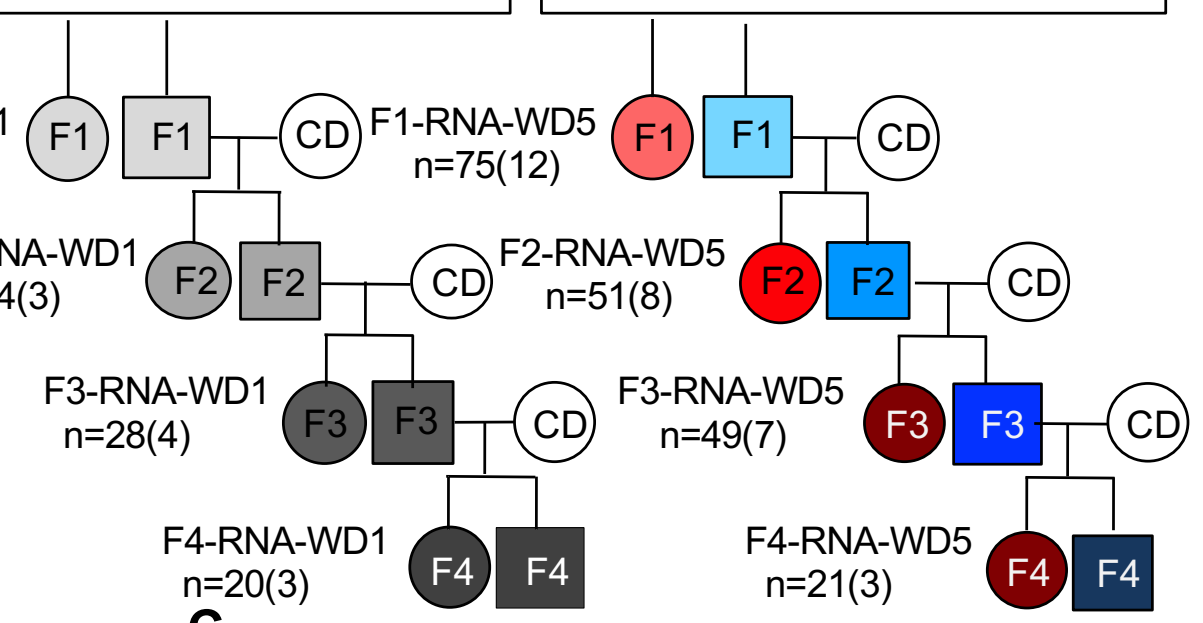

C
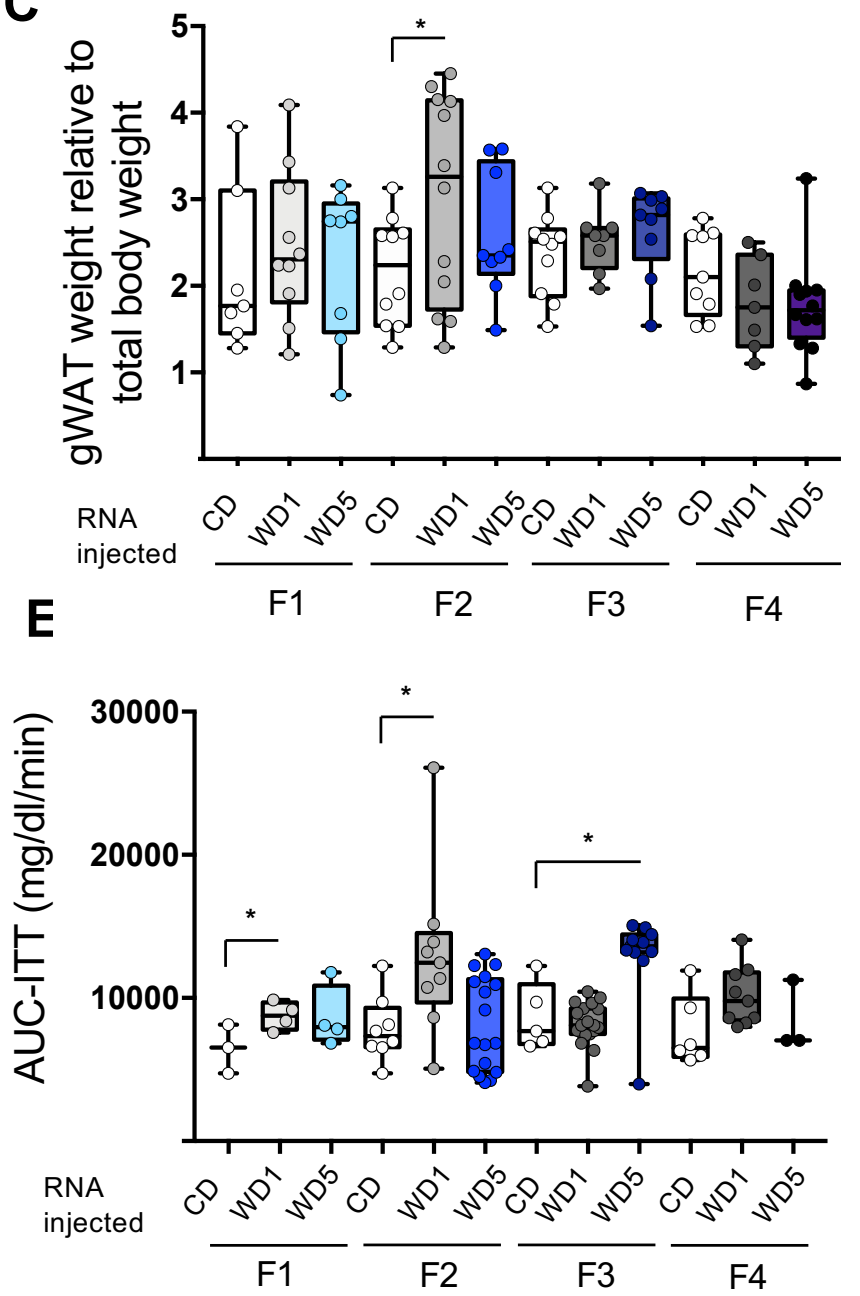

Figure 5 
(Father=5)

(Litter=5)

$(n=15)$

WD2

(Father=4)

(Litter=6)

$(n=15)$

WD3

(Father=8)

(Litter=8)

$(n=17)$

WD4

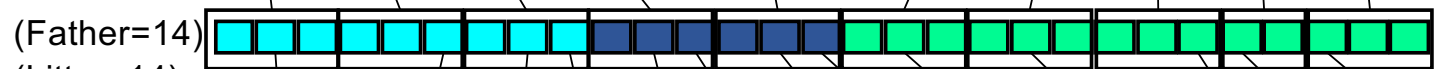

(Litter=14)

$(\mathrm{n}=42)$

WD5

(Father=16)

(Litter=18)

$(n=53)$

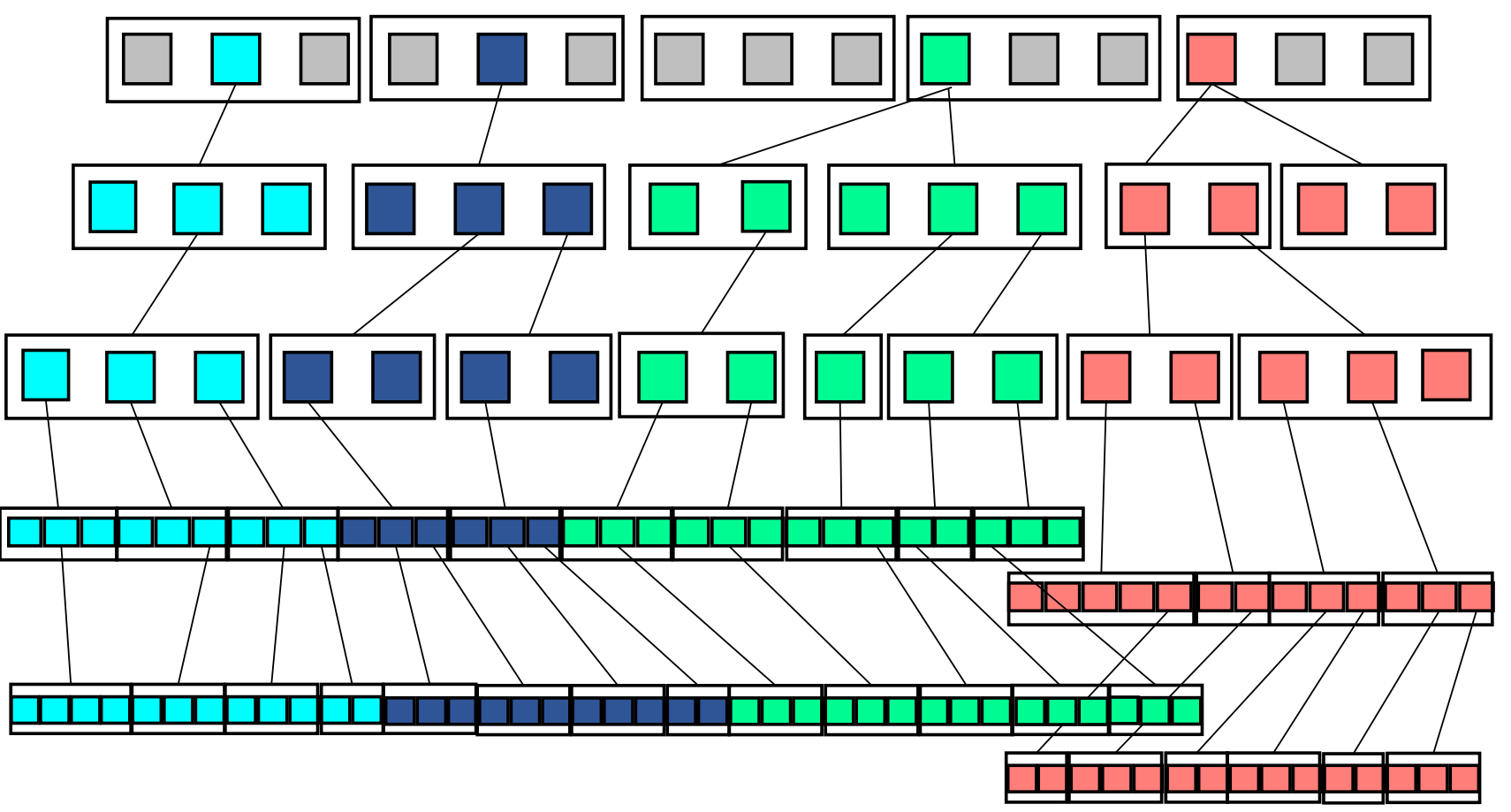

B

CD1

(Father=3)

(Litter=5)

$(\mathrm{n}=10)$

CD2

(Father=4)

Litter=4)

$(n=8)$

CD3

(Father=2)

(Litter=2)

$(n=5)$

CD4

(Father=2)

(Litter=2)

$(n=4)$

CD5

(Father=4)

(Litter=4)

$(n=13)$
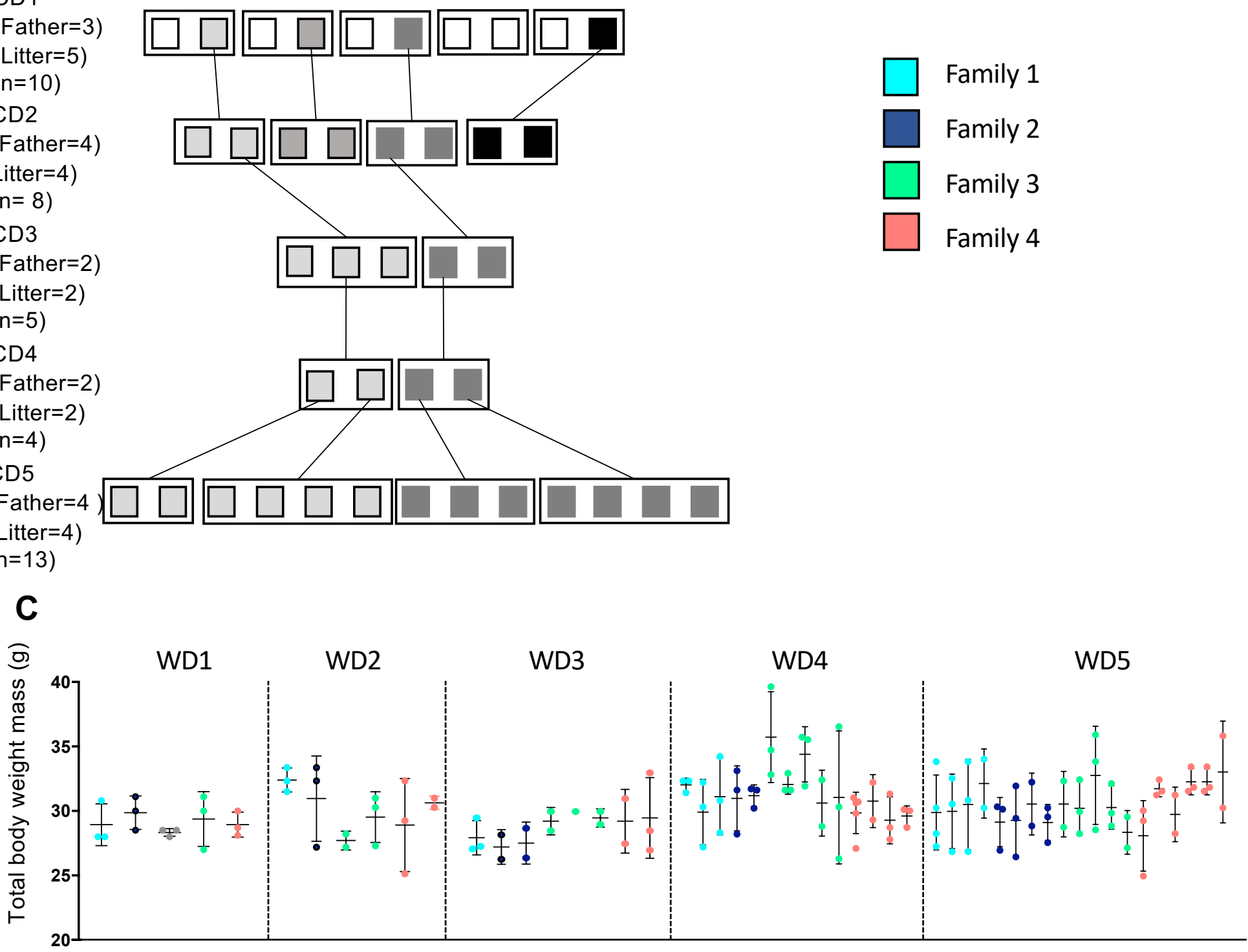
A

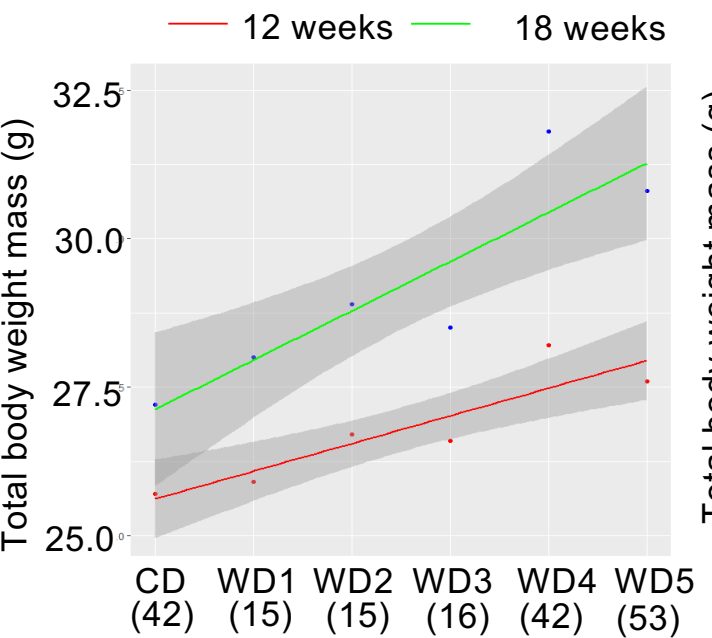

E

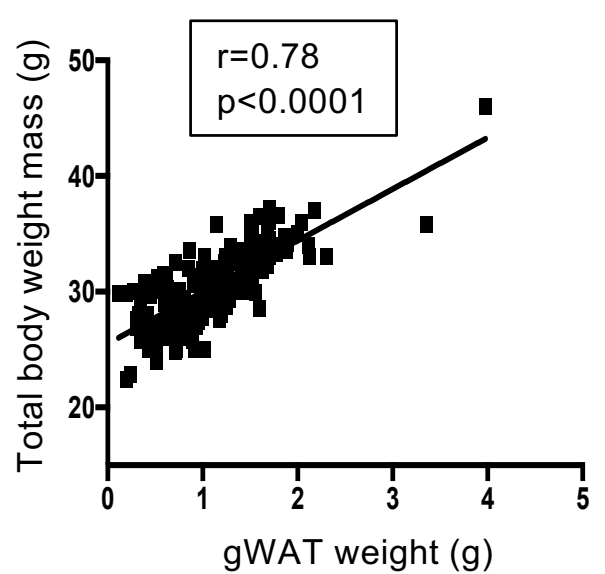

I

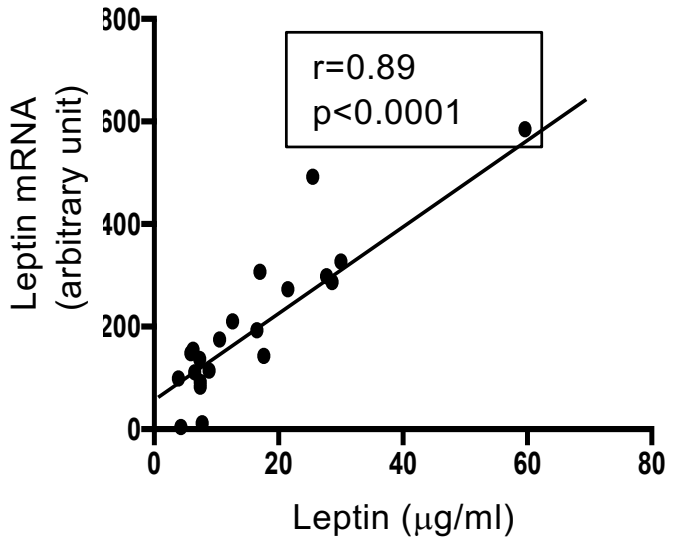

B
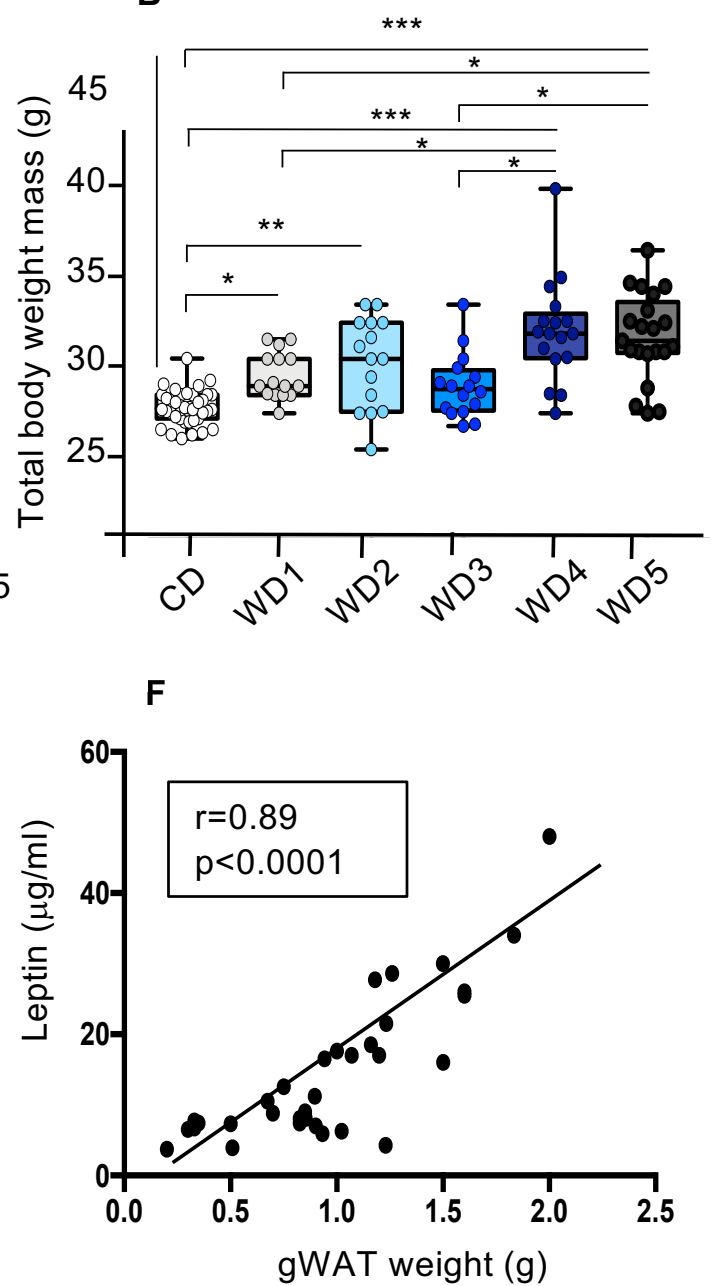

J

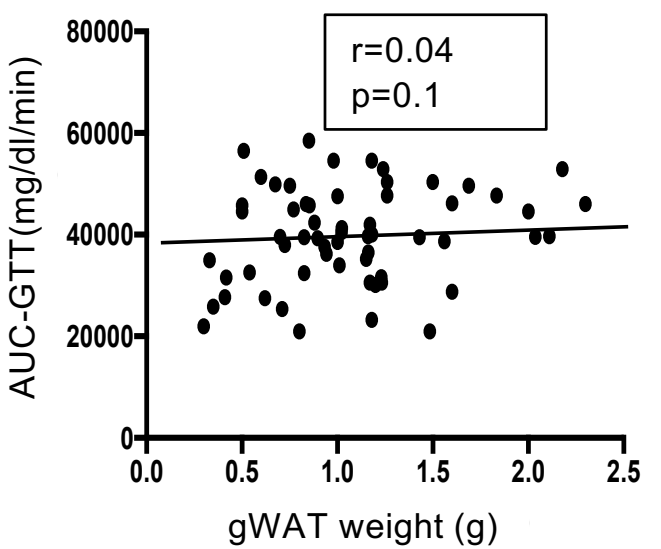

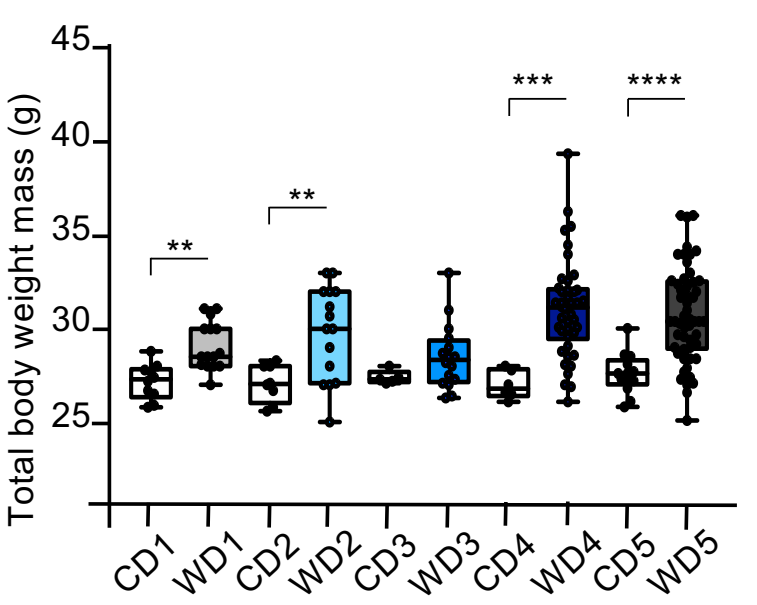

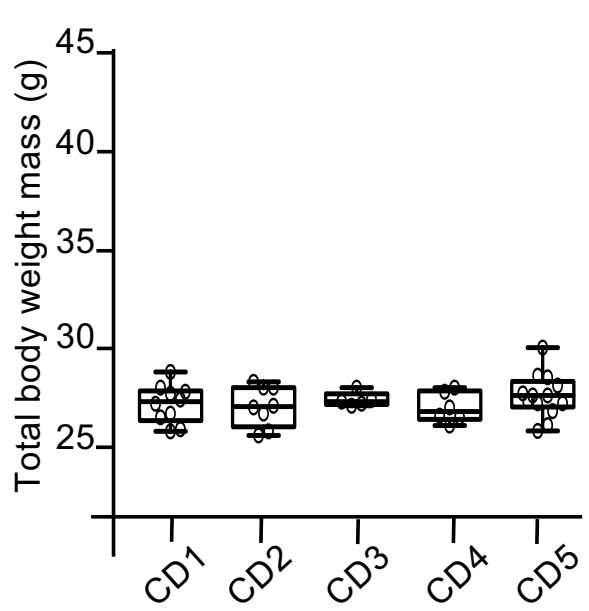

G

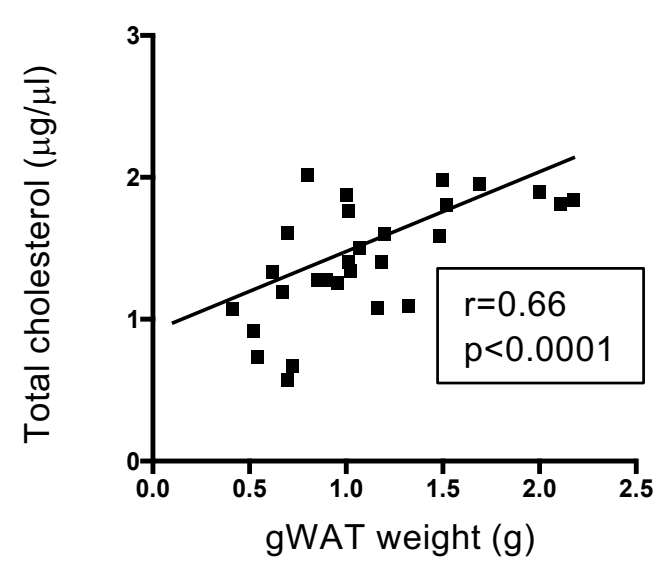

H

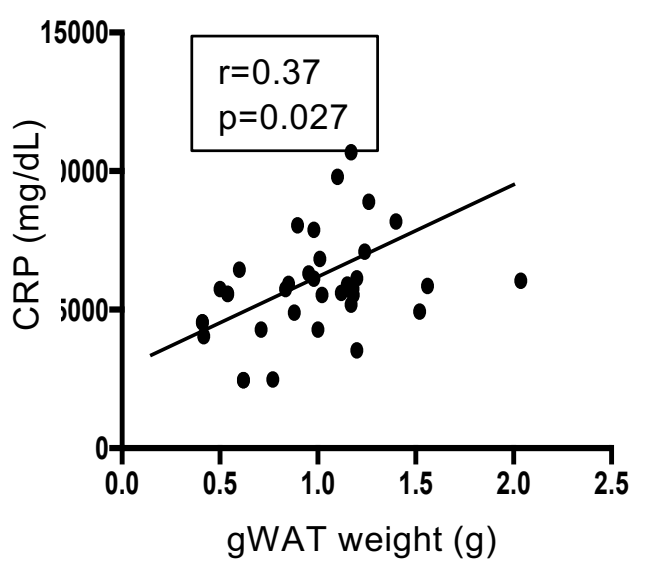




\section{Male Progenies}

A
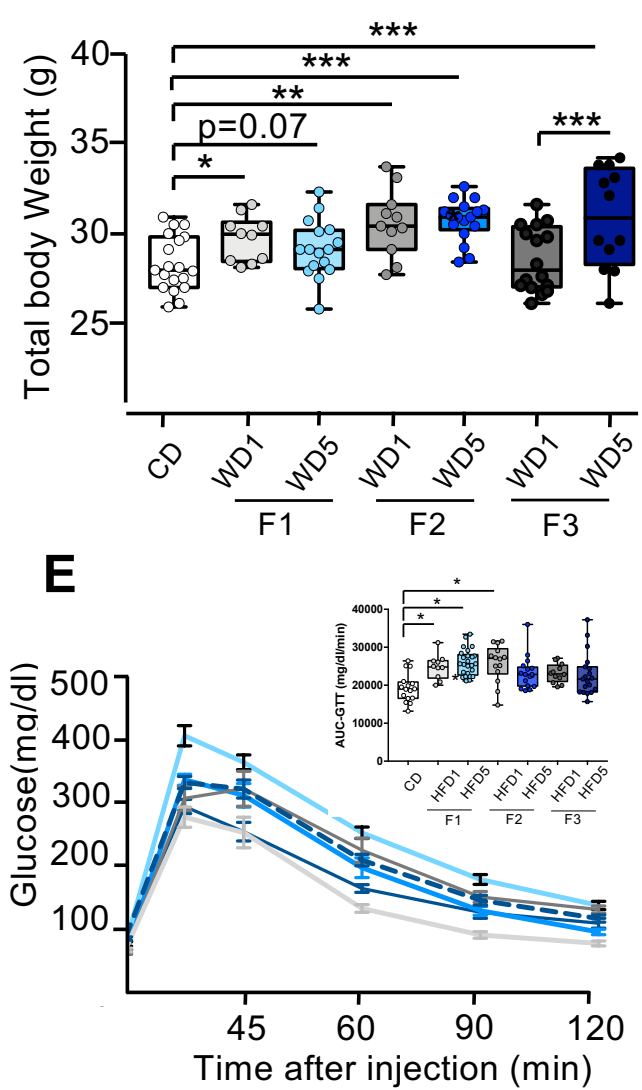

B

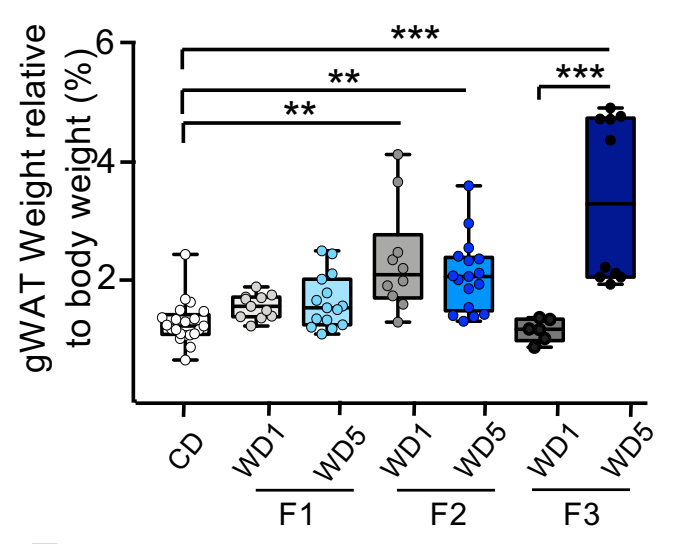

$\mathbf{F}$

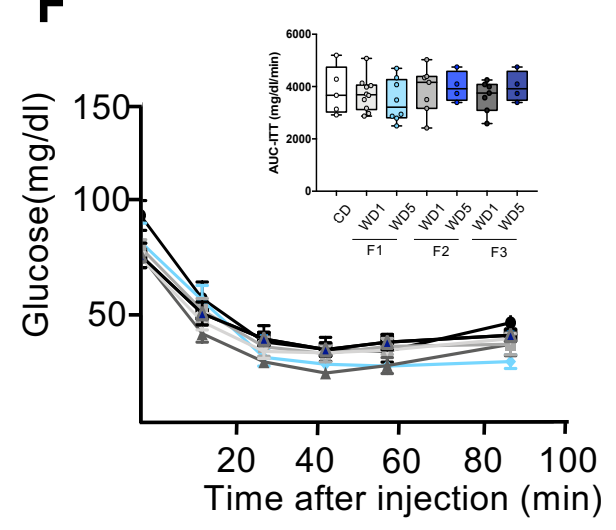

\section{Female Progenies}

C

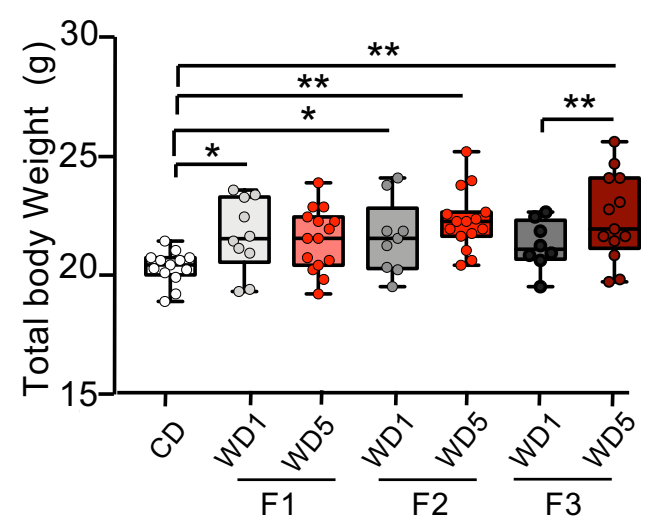

\section{G}

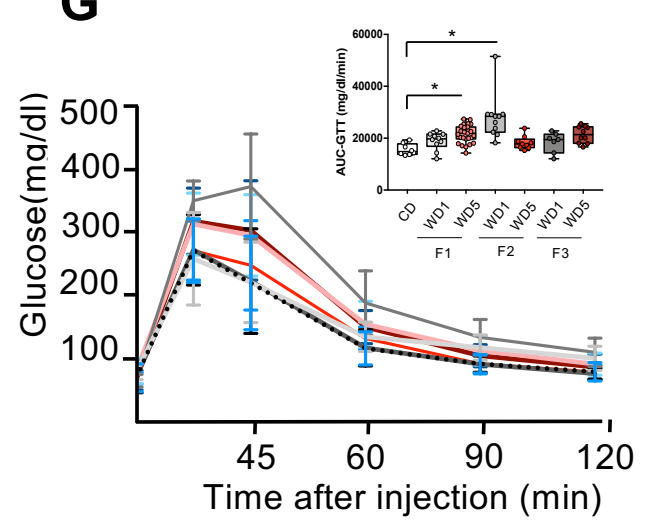

D

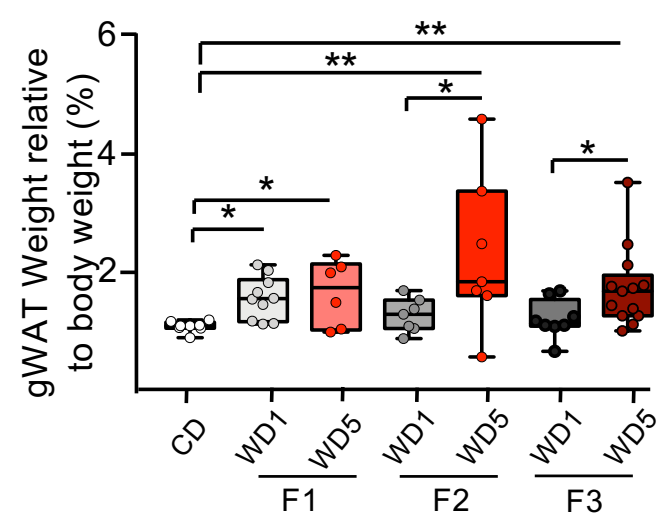

H

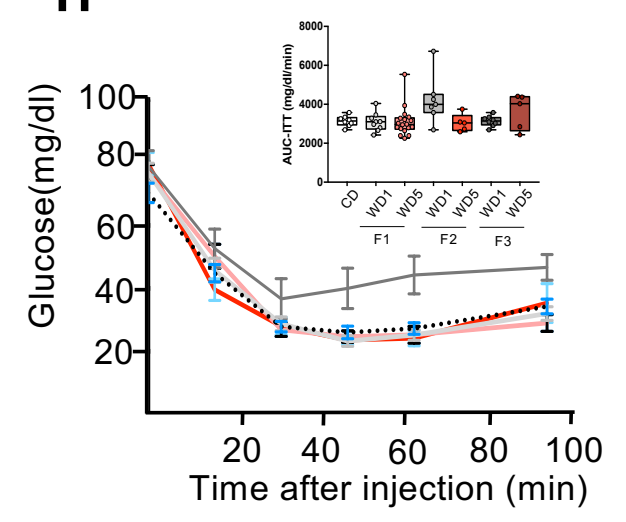


A

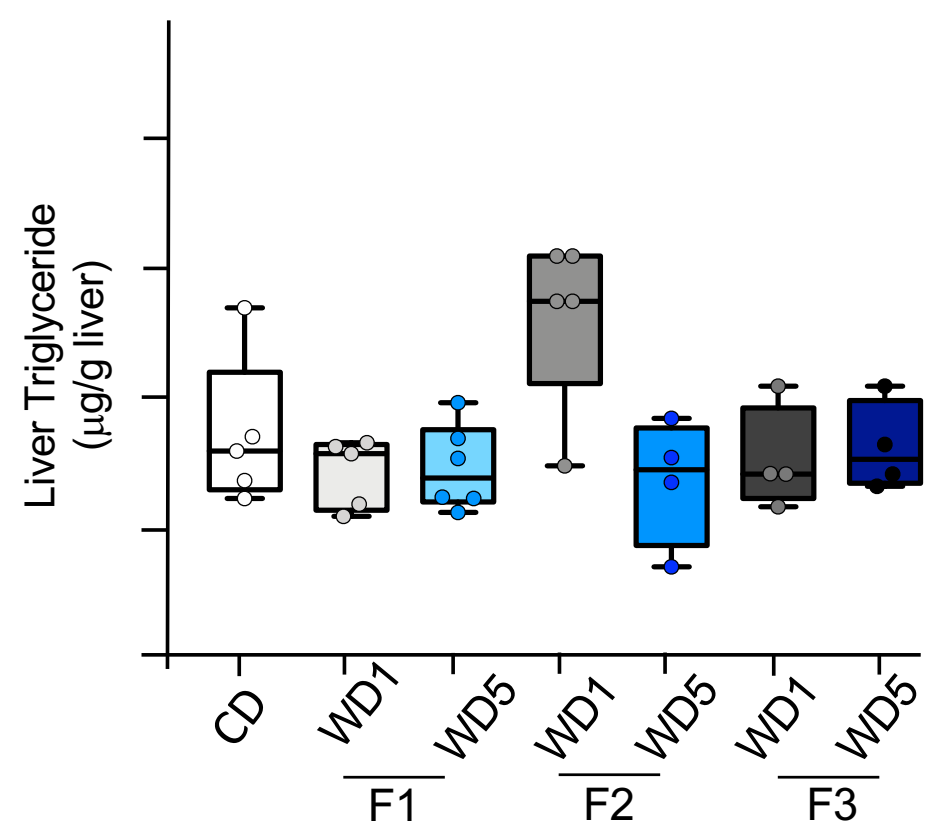

B

$C D$

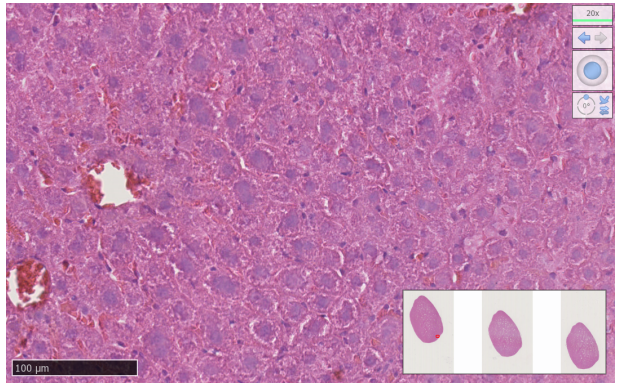

WD1 progenies
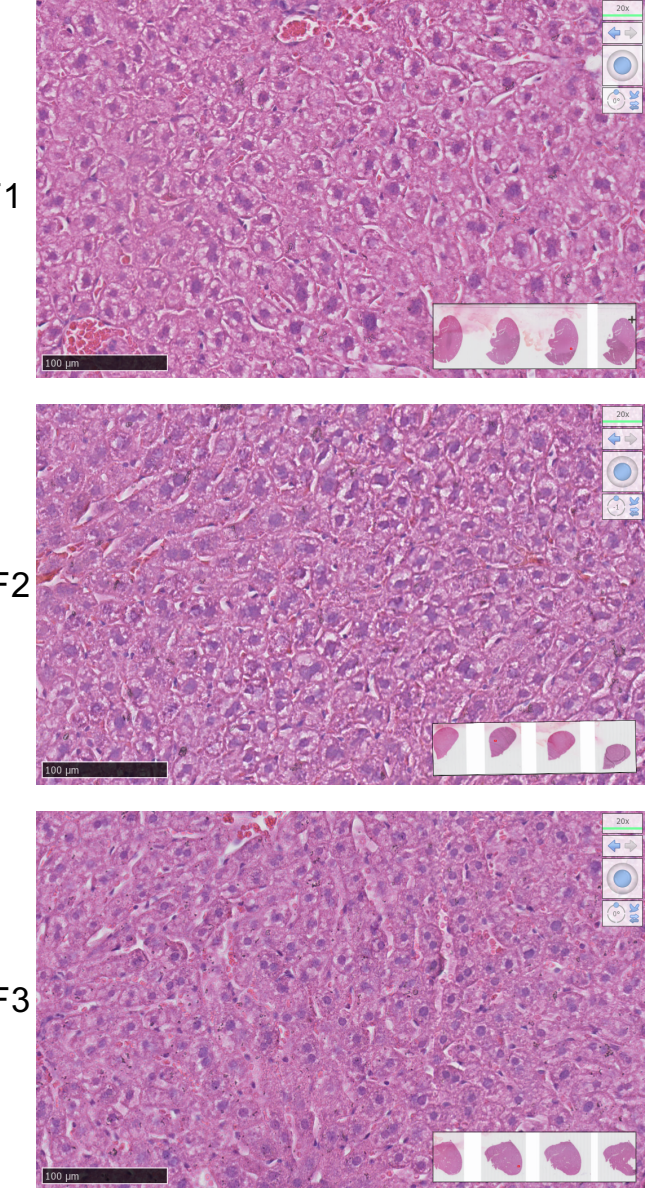

WD5 progenies
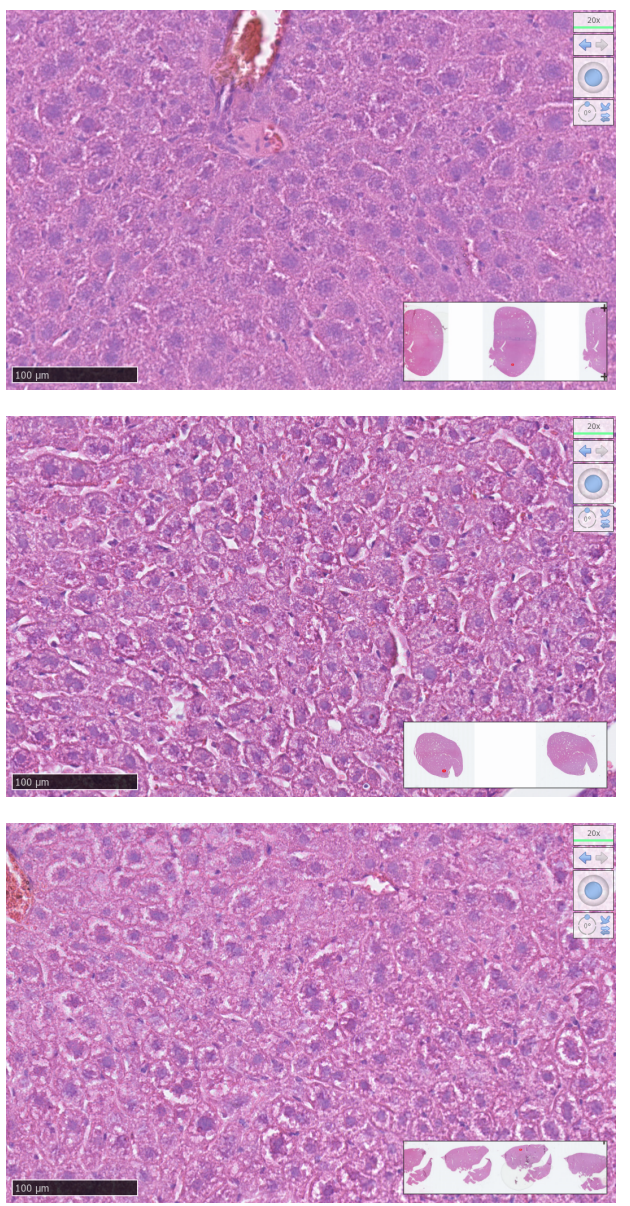

Figure 3-figure supplement 2 
A

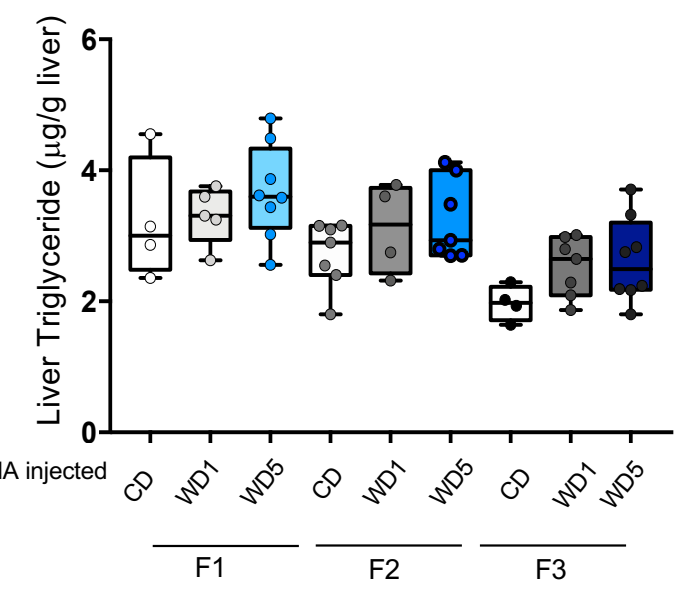

B

F1-RNA progenies

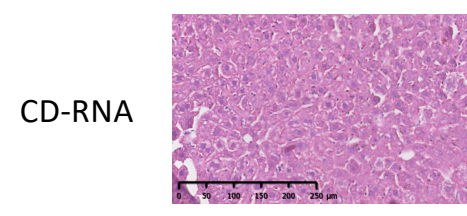

WD1-RNA

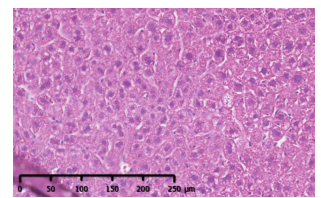

WD5-RNA

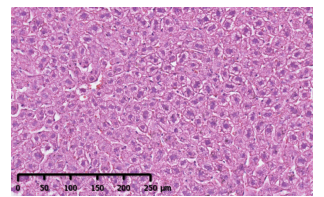

F2-RNA progenies
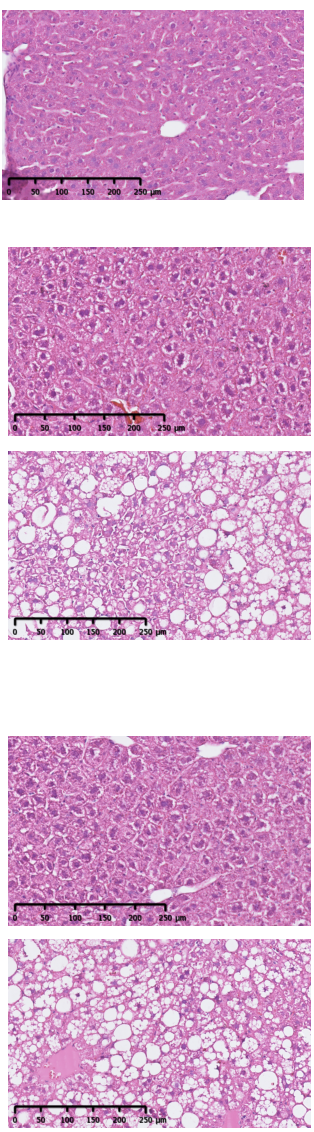

F3-RNA progenies
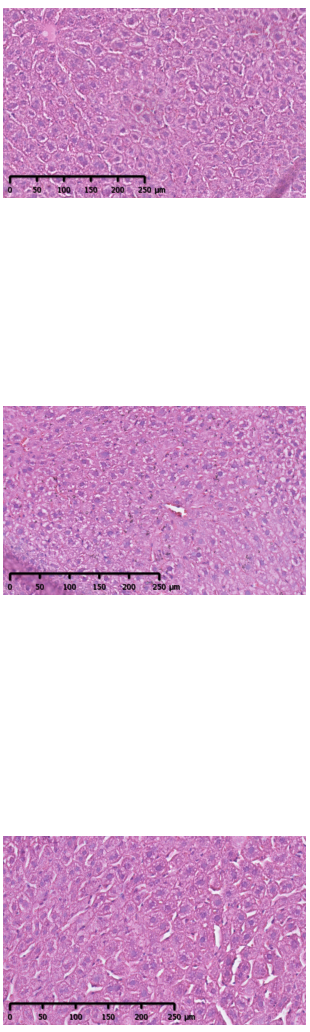